\title{
Soft ii-Open Sets in Soft Topological Spaces
}

\author{
Sabih W. Askandar, Amir A. Mohammed \\ Department of Mathematics, College of Education for Pure Sciences, University of Mosul, Mosul, Iraq \\ Email: sabihqaqos@gmail.com
}

How to cite this paper: Askandar, S.W. and Mohammed, A.A. (2020) Soft ii-Open Sets in Soft Topological Spaces. Open Access Library Journal, 7: e6308. https://doi.org/10.4236/oalib.1106308

Received: April 8, 2020

Accepted: May 16, 2020

Published: May 19, 2020

Copyright $\odot 2020$ by author(s) and Open Access Library Inc.

This work is licensed under the Creative Commons Attribution International License (CC BY 4.0).

http://creativecommons.org/licenses/by/4.0/

\begin{abstract}
In the current study, researchers have introduced modern concepts of soft open sets in the spaces of soft topologies named as soft i-open, soft inter-open and soft ii-open sets and they have explained the relations between these concepts and some other concepts such as the soft semi-open and soft $\alpha$-open sets. They have also used a definition of soft open neighborhood of a point in $X$ to introduce and discuss some applications of soft ii-open sets as soft ii-closure, soft ii-interior, soft ii-exterior, soft ii-border and soft ii-frontier of the soft set. Finally, they gave an example to explain and clarify all mentioned applications.
\end{abstract}

\section{Subject Areas}

Topology

\section{Keywords}

Soft ii-Open Sets, Soft i-Open Sets, Soft Inter-Open Sets

\section{Introduction}

The notion of soft set was proposed by Molodtsov, in 1999 (see [1]). After that, the same researcher has applied the concept theory on many different fields and various directions with many other researchers (see [2] [3] [4] [5]). In 2011 and 2012 the notion of soft topological spaces over an initial universe, was studied by many researches (see [6]-[12]). It is known that soft topology is a modern and crucial disciplinary has the ability to the improvement of creative and innovative approaches and new mathematical models that participate in finding solutions of complicated issues in many important sciences. Throughout this study, $(X, \tau$, $E$ ) always denotes soft topological space. The concept and the notion of i-open sets in the topological spaces ordinary type were presented by Mohammed, A.A. and Askandar, S.W. in 2012 [13]. These researchers managed to entire these sets 
together with various generalized open sets concepts. After that, in 2016, the aforementioned results were generalized through the utilization of i-open sets' notion to present separation axioms i-separation axioms like $T_{\mathrm{oi}}, T_{1 \mathrm{i}}$ and $T_{2 \mathrm{i}}$. Also, the researchers managed to find the connection between soft i-separation axioms and different notions like semi-separation axioms, and $\alpha$-separation axioms. Furthermore, the sets of i-star generalized w-closed and i-open in bitopological spaces $\left(X, \tau_{1}, \tau_{2}\right)$ were defined through the utilization of i-open sets the definition which exists in topological space. Also various fundamental relations and properties among these types of groups were introduced. Moreover, the closed sets notion was generalized, by the researchers, to i-generalized closed sets aided by i-open sets and gained topological properties and presented two types of maps known as gi-homeomorphisms and ig-homeomorphisms by examining their properties. However, in this paper, the researches utilize the definitions of i-open sets, $\alpha$-open sets [14] and semi-open sets [15]. Within the part two 2, the researchers present famous basic concepts and findings of soft topological space and the soft sets' theory. In Section 3 they will introduce and study soft inter-open, soft i-open and soft ii-open sets' definitions; then the researchers find its place among some other types of soft open sets as soft semi-open (see [16]) and sets of soft $\alpha$-open (see [17]) in the soft topological spaces (see Theorems 3.2, 3.5, 3.7, 3.11, 3.12, 3.15, Corollaries 3.4, 3.9, 3.17, 3.20). In Section 4, they initiate and analyze some applications of soft ii-closure, soft ii-open sets as soft ii-interior, soft ii-border, soft ii-frontier and soft ii-exterior of soft sets within the spaces of soft topologies.

\section{Preliminaries}

Definition 2.1: Consider $(X, \tau)$ as the topological space. A subset $A \subseteq X$ will be:

1) [14]. $\alpha$-open whether $A \subseteq \operatorname{Int}(\operatorname{Cl}(\operatorname{Int}(A)))$.

2) [15]. Semi-open whether:

a) $A \subseteq C l(\operatorname{Int}(A))$.

b) If $\exists G \in \tau, G \neq \varnothing, X$ such that $G \subseteq A \subseteq C l(G)$.

3) [18]. Inter-open or in short (int-open set) if $\exists G \in \tau, G \neq \varnothing, X$ such that $\operatorname{Int}(A)=G$.

4) [13]. i-open if $\exists G \in \tau, G \neq \varnothing, X$ such that $A \subseteq C l(A \cap G)$.

5) [18]. ii-open if $\exists G \in \tau, G \neq \varnothing, X$ and satisfies the both conditions: a) $A \subseteq C l(A \cap G)$. b) $\operatorname{Int}(A)=G$. In other words, a subset $A \subseteq X$ is an ii-open if it is inter-open and i-open set together.

Definition 2.2: [1]. Consider $X$ to be a universe' initial, then $P(X)$ denotes the $X$ set of power; $E$ is a parameters set and $(\varnothing \neq A \subseteq E)$. A couple $(K, A)$ or $K_{A}$ to be named as a soft set over $X$, wherein $K$ considered as mapping $K: A \rightarrow P(X)$. Over $X$ soft set is parameterized collection of subdivisions of $X$. in Specific $e \in A, K(e)$ considered the group of e-is to be soft set $(K, A)$ group of e-approximate factors, whether " $e \notin A$, so $K(e)=\varnothing$ i.e. 
$K_{A}=\{K(e): e \in A \subseteq E, K: A \rightarrow P(X)\} " . S S\left(X_{A}\right)$ denotes all these soft sets over $X$ family.

Definition 2.3: [5]. Consider $K_{A}, L_{B} \tilde{\in} S S\left(X_{E}\right)$. Then $K_{A}$ is a soft subset of $L_{B}$, denoted by $K_{A} \subseteq L_{B}$, whether 1) $A \subseteq B$.2) $K(e) \subseteq L(e), \forall e \in A$.

Definition 2.4: [5]. $K_{A}, L_{B} \tilde{\in} S S\left(X_{E}\right)$ are said to be soft equal if $K_{A} \tilde{\subseteq} L_{B}$ and $L_{B} \tilde{\subseteq} K_{A} L_{B}$.

Definition 2.5: [4]. The complement of $(K, A)$ soft set is designated by $(K$, $A)^{c}$, is determined by $(K, A)^{c}=\left(K^{c}, A\right), K^{c}: A \rightarrow P(X)$ is a mapping introduced by $K^{c}(e)=X-K(e)$, The $(K)$ soft complement function is $\forall e \in A$ and $K^{c}$. Apparently, $\left((K, A)^{c}\right)^{c}=(K, A)$.

Definition 2.6: [12]. The difference of $(K, E)$ and $(L, E)$ as soft sets in $X$, indicated by $(K, E) \backslash \tilde{(}(L, E)$ is the soft set $(M, E)$ wherein, $\forall e \in A$, $M(e)=K(e) \backslash L(e)$.

Definition 2.7: [12]. Consider $(K, E)$ is a soft set over $x \in X$ and $X$. It can be said that $x \tilde{\in}(K, E)$ whether $x \in K(e), \forall e \in A$.

Definition 2.8: [12]. Consider $x \in X$, as a soft set $(x, E)$ over $X$, wherein $x_{E}(e)=\{x\}, \forall e \in E$ is denoted by $x_{E}$ and was addressed as the single consider on soft point.

Definition 2.9: [5]. ( $K, A)$, as a soft set, over $X$ is:

1) A null soft set indicated by $\phi_{A}$ or $\phi$ whether $\forall e \in A, K(e)=\phi$.

2) An absolute soft set indicated by $\tilde{A}$ or $X_{A}$ if $\forall e \in A, K(e)=X$. Clearly $X_{A}^{C}=\phi_{A}$ and $\phi_{A}^{C}=X_{A}$.

Definition 2.10: [12]. A soft set $(K, E) \tilde{\in} S S\left(X_{E}\right)$ named as a soft point in $X_{E}$ is indicated by $K\left(e^{c}\right) \neq \phi \quad \forall e^{c} \in E-\{e\}$, and $e_{K}$ if $\exists x \in X$ and $e \in E$, $K(e) \neq \phi$. The soft point $e_{K}$ belongs to the soft set $(G, E), e_{K} \tilde{\in}(G, E)$, whether regarding the factor $e \in E, e_{K} \subseteq G(e)$. The group of $X$ whole soft points is indicated by $S P(X)$.

Definition 2.11: [5]. The soft set $(M, C)$ is the result of the union of both soft sets $(L, B)$ and $(K, A)$ over $X$ wherein $C=A \cup B \quad \forall e \in C$ and

$$
M(e)=\left\{\begin{array}{l}
K(e), e \in A \backslash B \\
L(e), e \in B \backslash A \\
K(e) \cup L(e), e \in A \cap B
\end{array}\right.
$$

Definition 2.12: [5]. The soft set $(M, C)$ is result of the intersection of both soft sets $(L, B)$ and $(K, A)$ on $X$ is wherein $C=A \cap B, \forall e \in C$, $M(e)=K(e) \cap L(e)$.

Definition 2.13: [19]. Consider $I$ is an random indexed group and $L=\left\{\left(K_{i}, E\right), i \in I\right\}$ is subdivision family of $S S\left(X_{E}\right)$.

1) $(M, E)$ considered as the soft set of $L$ union wherein $M(e)=\bigcup_{i \in I} K_{i}(e)$ $\forall e \in E, \bigcup_{i \in I}\left(K_{i}, E\right)=(M, E)$.

2) $(N, E)$ considered as the soft set of $L$ crossroad wherein $N(e)=\bigcap_{i \in I} K_{i}(e)$ $\forall e \in E, \bigcap_{i \in I}\left(K_{i} ; E\right)=(N, E)$.

Definition 2.14: [20]. Consider the set of attributes be $E$ and the universe is $X$. Therefore, the soft class which is indicated as $(X, E)$, is the compilation of whole 
soft sets over $X$ accompanied by attributes from $E$.

Proposition 2.15: [21]. Consider $(K, A),(L, A) \tilde{\in} S S\left(X_{A}\right)$ : therefore,

1) $\left.(K, A) \tilde{\bigcap}(K, A)^{c}=\phi_{A} .2\right)(K, A) \tilde{\bigcap}(L, A)=\phi_{A}$ whether and whether $(K, A) \tilde{\subseteq}(L, A)^{c}$ and $\left.(L, A) \subseteq(K, A)^{c} .3\right)(K, A) \tilde{\subseteq}(L, A)$ if and only if $(L, A)^{c} \tilde{\subseteq}(K, A)^{c}$.

Proposition 2.16: [11]. Consider $(K, A),(L, A) \tilde{\in} S S\left(X_{A}\right)$. Then

1) $\left.((K, A) \tilde{U}(L, A))^{c}=(K, A)^{c} \tilde{U}(L, A)^{c} .2\right)$ $((K, A) \tilde{\cap}(L, A))^{c}=(K, A)^{c} \tilde{\cap}(L, A)^{c}$.

Definition 2.17: [11]. The group of soft sets over $X$ is " $\tau$, the soft topology on $X$ is $\tau$ whether:

1) $\varnothing_{E}, X_{E}$ comes from to $\tau$. 2) $\tau$ Owes all the union of number soft sets of $\tau$ 3) Also, any 2 soft sets in $\tau$ intersection belong to $\tau$. The soft topological space known as the triple consider $(X, \tau, E)$. Soft open sets are known as the representative of $\tau$. If the complement $(K, E)^{c}$ of $(K, E)$ in $(X, \tau, E)$ belongs to $\tau$, then the soft set called a soft closed set. $\tau^{\mathcal{E}}$ denotes the set of the whole soft closed sets over $X$.

Definition 2.18: Consider $(K, E)$ be a soft set in $(X, \tau, E)$. Therefore,

1) [11]. The intersection of the whole soft closed sets including $(K, E)$ is the soft closure of $(K, E)$ which is indicated by $C l(K, E)$. Containing $(K, E)$, the lowest soft closed set is $C l(K, E)$.

$2)$ [8]. The union of the whole soft open sets which are contained in $(K, E)$ is the $(K, E)$ soft interior which is indicated by $\operatorname{Int}(K, E)$. Therefore, the largest soft open set contained in $(K, E)$ is $\operatorname{Int}(K, E)$.

Theorem 2.19: [8]. Consider $(K, E)$ be a soft set in $(X, \tau, E)$. Then

1) $\left.\left.\operatorname{Int}(K, E)^{c}=(C l(K, E))^{c} .2\right) \operatorname{Cl}(K, E)^{c}=(\operatorname{Int}(K, E))^{c} .3\right)$ $\operatorname{Int}(K, E)=\left(C l(K, E)^{c}\right)^{c}$.

\section{Soft ii-Open Sets in the Soft Topological Spaces}

Definition 3.1: Consider $(F, E)$ as a soft set in $(X, \tau, E)$, therefore, $(F, E)$ is said to be,

1) If there is a soft open set $(G, E) \neq \varnothing, X$ where $\operatorname{Int}(F, E)=(G, E)$, then $(F, E)$ is soft Inter-open (in short soft Int-open).

2) Soft i-open whether there is a soft open set $(G, E) \neq \varnothing, X$ where $(F, E) \tilde{\subseteq} C l((F, E) \tilde{\cap}(G, E))$.

3) Soft ii-open whether there is a soft open set $\varnothing, X \neq(G, E)$ where:

a) $(F, E) \tilde{\subseteq} C l((F, E) \tilde{\cap}(G, E))$.

b) $\operatorname{Int}(F, E)=(G, E)$.

4) $[16]$. Soft semi-open if:

a) $(F, E) \tilde{\subseteq} C l(\operatorname{Int}(F, E))$.

b) Whether a soft open set exists $(G, E) \neq \varnothing, X$ where $(G, E) \tilde{\subseteq}(F, E) \tilde{\subseteq} C l(G, E)$.

5) [17]. Soft $\alpha$-open if $(F, E) \tilde{\subseteq} \operatorname{Int}(C l(\operatorname{Int}(F, E)))$.

The complement of soft ii-open (resp., soft $\alpha$-open, soft i-open, soft int-open, and soft semi-open) sets known as soft ii-closed (resp., soft int-closed, soft i-closed, 
soft semi-closed and soft $\alpha$-closed) sets. The intersection of all soft ii-closed (resp., soft int-closed, soft i-closed, soft semi-closed and soft $\alpha$-closed) sets over $X$ containing $(F, E)$ is called the soft ii-closure (resp., soft int-closure, soft i-closure, soft semi-closure and soft $\alpha$-closure) of $(F, E)$ and denoted by ii- $C l(F$, $E)$ (resp., int- $C l(F, E)$, i- $C l(F, E), s-C I(F, E)$ and $\alpha-C I(F, E)$ ).

The union of whole soft ii-open (resp., int-open, i-open, semi-open and $\alpha$-open) sets over $X$ contained in $(F, E)$ known as a soft ii-interior (resp., int-interior, i-interior, semi-interior and $\alpha$-interior) of a soft set $(F, E)$ and indicated by ii-Int $(F, E)$ (resp., int-Int $(F, E)$, i-Int $(F, E), s$-Int $(F, E)$ and $\alpha-\operatorname{Int}(F, E))$. The group of whole soft open (resp., ii-open, int-open, i-open, semi-open and $\alpha$-open sets),(soft closed, ii-closed, int-closed, i-closed, semi-closed and $\alpha$-closed) sets in $(X, \tau, E)$ are indicated by $\operatorname{OS}\left(X_{E}\right)$ (resp., $\operatorname{IIOS}\left(X_{E}\right), \operatorname{INTOS}\left(X_{E}\right), \operatorname{IOS}\left(X_{E}\right), \operatorname{SOS}\left(X_{E}\right)$, $\alpha \operatorname{OS}\left(X_{E}\right), \operatorname{CS}\left(X_{E}\right), \operatorname{IICS}\left(X_{E}\right), \operatorname{INTCS}\left(X_{E}\right), \operatorname{ICS}\left(X_{E}\right), S C S\left(X_{E}\right)$ and $\left.\alpha \operatorname{CS}\left(X_{E}\right)\right)$.

Theorem 3.2: Each soft open set is a soft i-open.

Proof: Consider $(G, E)$ as soft open set in $(X, \tau, E)$. It is clear that $(G, E) \tilde{\subseteq} C l((G, E) \tilde{\cap}(G, E))$, then, $(G, E) \tilde{\subseteq} C l(G, E)$. Hence $(G, E)$ is a soft i-open.

Example 3.3: Consider $X=\left\{x_{1}, x_{2}, x_{3}\right\}, \tau=\left\{\phi_{E},\left(F_{1}, E\right),\left(F_{2}, E\right), X_{E}\right\}$, $E=\left\{e_{1}, e_{2}\right\}$.where $\left(F_{1}, E\right)=\left\{\left(e_{1},\left\{x_{1}\right\}\right),\left(e_{2},\left\{x_{1}\right\}\right)\right\}$, $\left(F_{2}, E\right)=\left\{\left(e_{1},\left\{x_{1}, x_{3}\right\}\right),\left(e_{2},\left\{x_{1}, x_{3}\right\}\right)\right\}$.

Consider, " $(F, E)=\left\{\left(e_{1},\left\{x_{1}, x_{2}\right\}\right),\left(e_{2},\left\{x_{1}, x_{2}\right\}\right)\right\}$ ".

$$
\begin{aligned}
C S\left(X_{E}\right)= & \left\{X_{E},\left(F_{1}, E\right)^{c}=\left\{\left(e_{1},\left\{x_{2}, x_{3}\right\}\right),\left(e_{2},\left\{x_{2}, x_{3}\right\}\right)\right\},\right. \\
& \left.\left(F_{2}, E\right)^{c}=\left\{\left(e_{1},\left\{x_{2}\right\}\right),\left(e_{2},\left\{x_{2}\right\}\right)\right\}, \phi_{E}\right\}
\end{aligned}
$$

Apparently, $(F, E)$ is a soft $\mathrm{i}$-open set due to the existence a soft open set $(G, E)=\left(F_{1}, E\right)$ where $(G, E) \tilde{\subseteq} C l((F, E) \tilde{\cap}(G, E))$, yet $(F, E)$ is not soft open.

Corollary 3.4: Every soft closed set is a soft i-closed.

In example (3.3), it is obvious that $(F, E)=\left\{\left(e_{1},\left\{x_{3}\right\}\right),\left(e_{2},\left\{x_{3}\right\}\right)\right\}$ is a soft i-closed set but is not soft closed.

Theorem 3.5: Each soft semi-open set is a soft $\mathrm{i}$-open.

Proof: Consider $(F, E)$ as a soft semi-open set in $(X, \tau, E)$. by Definition (3.1)

(4) (b) there exist a soft open set $(G, E)$ where

$$
(G, E) \tilde{\subseteq}(F, E) \tilde{\subseteq} C l(G, E)
$$

Since $(G, E) \tilde{\subseteq}(F, E)$, then

$$
(F, E) \tilde{\bigcap}(G, E)=(G, E)
$$

By (1) and (2) there is, $(F, E) \tilde{\subseteq} C l((F, E) \tilde{\cap}(G, E))$. Therefore, $(F, E)$ considered as a soft $\mathrm{i}$-open set.

Example 3.6: Consider $X=\left\{x_{1}, x_{2}, x_{3}\right\}, \tau=\left\{\phi_{E},\left(F_{1}, E\right), X_{E}\right\}, E=\left\{e_{1}, e_{2}\right\}$.

Such that, " $\left(F_{1}, E\right)=\left\{\left(e_{1},\left\{x_{1}, x_{2}\right\}\right),\left(e_{2},\left\{x_{1}, x_{2}\right\}\right)\right\}$ ”.

Consider $(F, E)=\left\{\left(e_{1},\left\{x_{1}, x_{3}\right\}\right),\left(e_{2},\left\{x_{1}, x_{3}\right\}\right)\right\}$. 


$$
\begin{gathered}
O S\left(X_{E}\right)=\left\{\phi_{E},\left(F_{1}, E\right), X_{E}\right\}, \\
C S\left(X_{E}\right)=\left\{X_{E},\left(F_{1}, E\right)^{c}=\left\{\left(e_{1},\left\{x_{3}\right\}\right),\left(e_{2},\left\{x_{3}\right\}\right)\right\}, \phi_{E}\right\} .
\end{gathered}
$$

Apparently $(F, E)$ has been considered as a soft i-open set because of the existence of a soft open set $(G, E)=\left(F_{1}, E\right)$ wherein $(F, E) \tilde{\subseteq} C l((F, E) \tilde{\cap}(G, E))$, but $(F, E)$ has not been considered soft semi-open.

Theorem 3.7: Each soft $\alpha$-open set is considered as a soft semi-open.

Proof: Consider $(F, E)$ as soft $\alpha$-open set in $(X, \tau, E)$. In the definitions (3.1) (5) it can be found;

“ $(F, E) \tilde{\subseteq} \operatorname{Int}(C l(\operatorname{Int}(F, E)))=\tilde{U}_{\mathrm{i}}\left(G_{\mathrm{i}}, E\right),\left(G_{\mathrm{i}}, E\right) \tilde{\subseteq C l}(\operatorname{Int}(F, E))$ ”, where $\left(G_{\mathrm{i}}, E\right)$ is a soft open set for all i. Then $(F, E) \simeq \operatorname{Cl}(\operatorname{Int}(F, E))$. Thus, $(F, E)$ is a soft semi-open set.

Example 3.8: Consider " $X=\left\{x_{1}, x_{2}, x_{3}, x_{4}\right\}$, $\tau=\left\{\phi_{E},\left(F_{1}, E\right),\left(F_{2}, E\right),\left(F_{3}, E\right), X_{E}\right\}, E=\left\{e_{1}, e_{2}\right\} ”$.

Such that $\left(F_{1}, E\right)=\left\{\left(e_{1},\left\{x_{1}\right\}\right),\left(e_{2},\left\{x_{1}\right\}\right)\right\}$, $\left(F_{2}, E\right)=\left\{\left(e_{1},\left\{x_{3}, x_{4}\right\}\right),\left(e_{2},\left\{x_{3}, x_{4}\right\}\right)\right\}$, $\left(F_{3}, E\right)=\left\{\left(e_{1},\left\{x_{1}, x_{3}, x_{4}\right\}\right),\left(e_{2},\left\{x_{1}, x_{3}, x_{4}\right\}\right)\right\}$.

Consider $(F, E)=\left\{\left(e_{1},\left\{x_{1}, x_{2}\right\}\right),\left(e_{2},\left\{x_{1}, x_{2}\right\}\right)\right\}$.

$$
\begin{aligned}
\operatorname{OS}\left(X_{E}\right)=\left\{\phi_{E},\left(F_{1}, E\right),\left(F_{2}, E\right),\left(F_{3}, E\right), X_{E}\right\}, \\
C S\left(X_{E}\right)=\left\{X_{E},\left(F_{1}, E\right)^{c}=\left\{\left(e_{1},\left\{x_{2}, x_{3}, x_{4}\right\}\right),\left(e_{2},\left\{x_{2}, x_{3}, x_{4}\right\}\right)\right\},\right. \\
\text { “ }\left(F_{2}, E\right)^{c}=\left\{\left(e_{1},\left\{x_{1}, x_{2}\right\}\right),\left(e_{2},\left\{x_{1}, x_{2}\right\}\right)\right\}, \\
\left.\left(F_{3}, E\right)^{c}=\left\{\left(e_{1},\left\{x_{2}\right\}\right),\left(e_{2},\left\{x_{2}\right\}\right)\right\}, \phi_{E}\right\}
\end{aligned}
$$

It is apparently that $(F, E)$ considered as a soft semi-open set, yet $(F, E)$ has not been considered a soft $\alpha$-open because $(F, E) \tilde{\not}\left(\operatorname{Int}(\operatorname{Cl}(\operatorname{Int}(F, E)))=\left(F_{1}, E\right)\right)$.

Corollary 3.9: Each soft $\alpha$-open set is a soft i-open.

Proof: Consider $(F, E)$ as a soft $\alpha$-open set in $(X, \tau, E)$. Based on the theory (3.7) it can be said; $(F, E)$ is a soft semi-open. Based on the theory (3.5) we can find $(F, E)$ is a soft $\mathrm{i}$-open set.

In Example (3.6), we see that $(F, E)=\left\{\left(e_{1},\left\{x_{1}, x_{3}\right\}\right),\left(e_{2},\left\{x_{1}, x_{3}\right\}\right)\right\}$ is a soft i-open set, but is not soft $\alpha$-open because $(F, E) \tilde{\not}\left(\operatorname{Int}(\operatorname{Cl}(\operatorname{Int}(F, E)))=\phi_{E}\right)$.

Corollary 3.10: By Theorems (3.2), (3.5) and (3.7), Corollary (3.9) we have the following diagram as shown in Figure 1.

Theorem 3.11: Each soft semi-open set is a soft ii-open.

Proof: Consider $(F, E)$ as a soft semi-open set in $(X, \tau, E)$. Based in the theory (3.5), it can be found that $(F, E)$ is a soft $\mathrm{i}$-open. Such that there is a soft open set $(G, E)$ where $(F, E) \tilde{\subseteq} C l((F, E) \tilde{\cap}(G, E))$. Now, we shall prove that $\operatorname{Int}(F, E)=(G, E)$. Note that if $\operatorname{Int}(F, E) \neq(G, E)$, for each soft open set $(G, E)$. Then, " $C l(\operatorname{Int}(F, E)) \neq C l(G, E)$ ". From above inclusions, it concludes that " $(F, E) \tilde{\subseteq} C l(\operatorname{Int}(F, E) \tilde{\cap}(F, E) \tilde{\cap}(G, E))$ ”. This means that $(F, E) \tilde{\mp} C l(G, E)$. This is a confliction. Thus, $\operatorname{Int}(F, E)=(G, E)$. Therefore, $(F, E)$ has been considered as a soft ii-open set. 


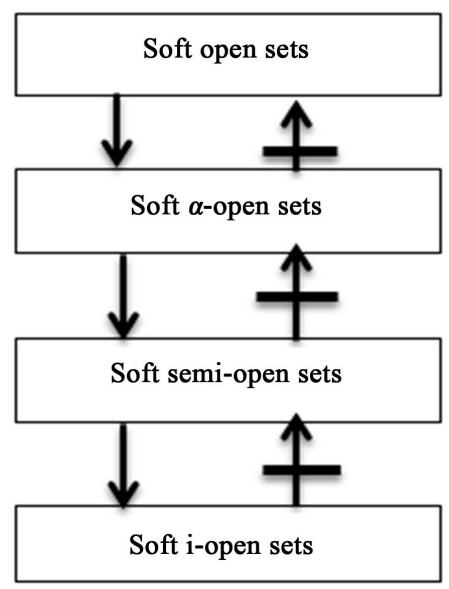

\section{Remarks}

1. (Soft open set $\longrightarrow$ Soft i-open set) means that each soft open set is a soft i-open set.

2. (Soft open set to be soft open set.

Figure 1. The relation among soft i-open, soft semi-open, soft open, and soft $\alpha$-open sets.

Theorem 3.12: Each soft open set is a soft ii-open.

Proof: Consider $(G, E)$ as a soft open set in $(X, \tau, E)$. Since

“ $(G, E) \tilde{\subseteq} C l((G, E) \tilde{\bigcap}(G, E))=C l(G, E)$ ”, it follows that $(G, E)$ is a soft i-open set. Also $\operatorname{Int}(G, E)=(G, E)$, we have $(G, E)$ is a soft Inter-open. Hence $(G, E)$ is a soft ii-open set.

Example 3.13: Consider $X=\left\{x_{1}, x_{2}, x_{3}\right\}, \tau=\left\{\phi_{E},\left(F_{1}, E\right),\left(F_{2}, E\right), X_{E}\right\}$, $E=\left\{e_{1}, e_{2}\right\}$.

Where $\left(F_{1}, E\right)=\left\{\left(e_{1},\left\{x_{2}\right\}\right),\left(e_{2},\left\{x_{2}\right\}\right)\right\},\left(F_{2}, E\right)=\left\{\left(e_{1},\left\{x_{2}, x_{3}\right\}\right),\left(e_{2},\left\{x_{2}, x_{3}\right\}\right)\right\}$. Consider $(F, E)=\left\{\left(e_{1},\left\{x_{1}, x_{2}\right\}\right),\left(e_{2},\left\{x_{1}, x_{2}\right\}\right)\right\}$.

$$
\begin{aligned}
& O S\left(X_{E}\right)=\left\{\phi_{E},\left(F_{1}, E\right),\left(F_{2}, E\right), X_{E}\right\}, \\
C S\left(X_{E}\right)=\{ & X_{E},\left(F_{1}, E\right)^{c}=\left\{\left(e_{1},\left\{x_{1}, x_{3}\right\}\right),\left(e_{2},\left\{x_{1}, x_{3}\right\}\right)\right\}, \\
& \left.\left(F_{2}, E\right)^{c}=\left\{\left(e_{1},\left\{x_{2}, x_{3}\right\}\right),\left(e_{2},\left\{x_{2}, x_{3}\right\}\right)\right\}, \phi_{E}\right\}
\end{aligned}
$$

Obviously $(F, E)$ considered as soft ii-open set, yet $(F, E)$ could not be a soft open.

Theorem 3.14: Whether $(X, \tau, E)$ considered as a soft topological space, therefore $\left(X, I I O\left(X_{E}\right), E\right)$ is also soft topological space.

Proof: Consider $(F, E)$ as a soft ii-open set, also consider $x \tilde{\in}(F, E)$. We will prove the existence of a soft open set, say $(G, E)$ wherein, $x \tilde{\in}(G, E) \tilde{\subseteq}(F, E)$. As $(F, E)$ considered as soft ii-open set, it pursues that it exists a soft open set $(G, E) \neq \phi, X$ where $(F, E) \tilde{\subseteq C l}((F, E) \tilde{\bigcap}(G, E))$ and $\operatorname{Int}(F, E)=(G, E)$. Therefore, $x \tilde{\in}(F, E)$ implies that $x \tilde{\in} C l(F, E)$ and $x \tilde{\in} C l(G, E)$. If $(G, E)$ considered as soft closed set, therefore, $x \tilde{\in}(G, E) \tilde{\subseteq}(F, E)$ and $(F, E)$ can be a soft open set, if $(G, E)$ is not a soft closed. Since $x \tilde{\in}(F, E)$ implies that 
$x \tilde{\epsilon}(C l(F, E) \tilde{\cap} C l(G, E))$ indicates that $x \tilde{\epsilon} C l(G, E)$. This creates an opposition. Thus, $(F, E)$ is soft open set. Hence, $I I O\left(X_{E}\right)$ satisfies the conditions of soft topology in the Definition (2.17). That means the triple $\left(X, I I O\left(X_{E}\right), E\right)$ can also be a soft topological space.

In Example (3.13), IIO $\left(X_{E}\right)=\left\{\phi_{E},\left(F_{1}, E\right),\left(F_{2}, E\right),(F, E), X\right\}$. It is clear that $(X, \tau, E)$ and $\left(X, I I O\left(X_{E}\right), E\right)$ can be a soft topological space.

Theorem 3.15: Each soft $\alpha$-open set is a soft ii-open.

Proof: Consider $(F, E)$ as a soft $\alpha$-open set in $(X, \tau, E)$. Since $(F, E) \tilde{\subseteq} \operatorname{Int}(C l(\operatorname{Int}(F, E))) \tilde{\subseteq} C l(\operatorname{Int}(F, E))$. Thus, $(F, E)$ can be soft semi-open. By Theorem (3.11) it can be found that $(F, E)$ can be taken as a soft ii-open

set.

Example 3.16: Consider $X=\left\{x_{1}, x_{2}, x_{3}, x_{4}\right\}$, $\tau=\left\{\phi_{E},\left(F_{1}, E\right),\left(F_{2}, E\right),\left(F_{3}, E\right), X_{E}\right\}, E=\left\{e_{1}, e_{2}\right\}$. Such that $\left(F_{1}, E\right)=\left\{\left(e_{1},\left\{x_{1}\right\}\right),\left(e_{2},\left\{x_{1}\right\}\right)\right\},\left(F_{2}, E\right)=\left\{\left(e_{1},\left\{x_{3}, x_{4}\right\}\right),\left(e_{2},\left\{x_{3}, x_{4}\right\}\right)\right\}$, $\left(F_{3}, E\right)=\left\{\left(e_{1},\left\{x_{1}, x_{3}, x_{4}\right\}\right),\left(e_{2},\left\{x_{1}, x_{3}, x_{4}\right\}\right)\right\}$. Consider $(F, E)=\left\{\left(e_{1},\left\{x_{2}, x_{3}, x_{4}\right\}\right),\left(e_{2},\left\{x_{1}, x_{3}, x_{4}\right\}\right)\right\}$. $\operatorname{OS}\left(X_{E}\right)=\left\{\phi_{E},\left(F_{1}, E\right),\left(F_{2}, E\right),\left(F_{3}, E\right), X_{E}\right\}$, $C S\left(X_{E}\right)=\left\{X_{E},\left(F_{1}, E\right)^{c}=\left\{\left(e_{1},\left\{x_{2}, x_{3}, x_{4}\right\}\right),\left(e_{2},\left\{x_{2}, x_{3}, x_{4}\right\}\right)\right\}\right.$,

$$
\begin{aligned}
& \left(F_{2}, E\right)^{c}=\left\{\left(e_{1},\left\{x_{1}, x_{2}\right\}\right),\left(e_{2},\left\{x_{1}, x_{2}\right\}\right)\right\}, \quad \text {. Obviously, }(F, E) \\
& \left.\left(F_{3}, E\right)^{c}=\left\{\left(e_{1},\left\{x_{2}\right\}\right),\left(e_{2},\left\{x_{2}\right\}\right)\right\}, \phi_{E}\right\}
\end{aligned}
$$

can be classified as a soft ii-open set not as a soft $\alpha$-open as $(F, E) \tilde{\varnothing} \operatorname{Int}\left(C l(\operatorname{Int}(F, E))=\left(F_{2}, E\right)\right)$.

Corollary 3.17: Each soft $\alpha$-open set is a soft inter-open.

Proof: Suppose that $(F, E)$ is a soft $\alpha$-open set. Since each soft $\alpha$-open set is a soft ii-open (Theorem 3.15) we have $(F, E)$ is a soft ii-open set, and since each soft ii-open set is a soft inter-open (Definition 3.1 (1 and 3)), we have, $(F, E)$ is a soft inter-open set.

Example 3.18: Consider $X=\left\{x_{1}, x_{2}, x_{3}\right\}, \tau=\left\{\phi_{E},\left(F_{1}, E\right),\left(F_{2}, E\right), X_{E}\right\}$, $E=\left\{e_{1}, e_{2}\right\}$. Such that " $\left(F_{1}, E\right)=\left\{\left(e_{1},\left\{x_{1}\right\}\right),\left(e_{2},\left\{x_{1}\right\}\right)\right\}$, $\left(F_{2}, E\right)=\left\{\left(e_{1},\left\{x_{2}, x_{3}\right\}\right),\left(e_{2},\left\{x_{2}, x_{3}\right\}\right)\right\}$ ”. Consider “ $(F, E)=\left\{\left(e_{1},\left\{x_{1}, x_{2}\right\}\right),\left(e_{2},\left\{x_{1}, x_{2}\right\}\right)\right\} " . O S\left(X_{E}\right)=\left\{\phi_{E},\left(F_{1}, E\right),\left(F_{2}, E\right), X_{E}\right\}$, “ $C S\left(X_{E}\right)=\left\{X_{E},\left(F_{1}, E\right)^{c}=\left\{\left(e_{1},\left\{x_{2}, x_{3}\right\}\right),\left(e_{2},\left\{x_{2}, x_{3}\right\}\right)\right\}\right.$, $\left.\left(F_{2}, E\right)^{c}=\left\{\left(e_{1},\left\{x_{1}\right\}\right),\left(e_{2},\left\{x_{1}\right\}\right)\right\}, \phi_{E}\right\}$ ." Obviously $(F, E)$ can't be soft $\alpha$-open but it is a soft inter-open, due to the fact that $(F, E) \tilde{\varnothing} \operatorname{Int}\left(C l(\operatorname{Int}(F, E))=\left(F_{1}, E\right)\right)$.

Remark 3.19: Each soft ii-open set is a soft i-open, soft inter-open, but the contrary is not true, as viewed in example (3.1.18), we see that $\left(F_{L}, E\right)=\left\{\left(e_{1},\left\{x_{2}\right\}\right),\left(e_{2},\left\{x_{2}\right\}\right)\right\}$ is not soft ii-open but is a soft i-open. Also $\left(F_{K}, E\right)=\left\{\left(e_{1},\left\{x_{1}, x_{2}\right\}\right),\left(e_{2},\left\{x_{1}, x_{2}\right\}\right)\right\}$ is not soft ii-open but a soft inter-open.

Corollary 3.20: By Corollaries (3.9) and (3.17), Theorems (3.11), (3.12), (3.15) we have the following diagram as shown in Figure 2. 


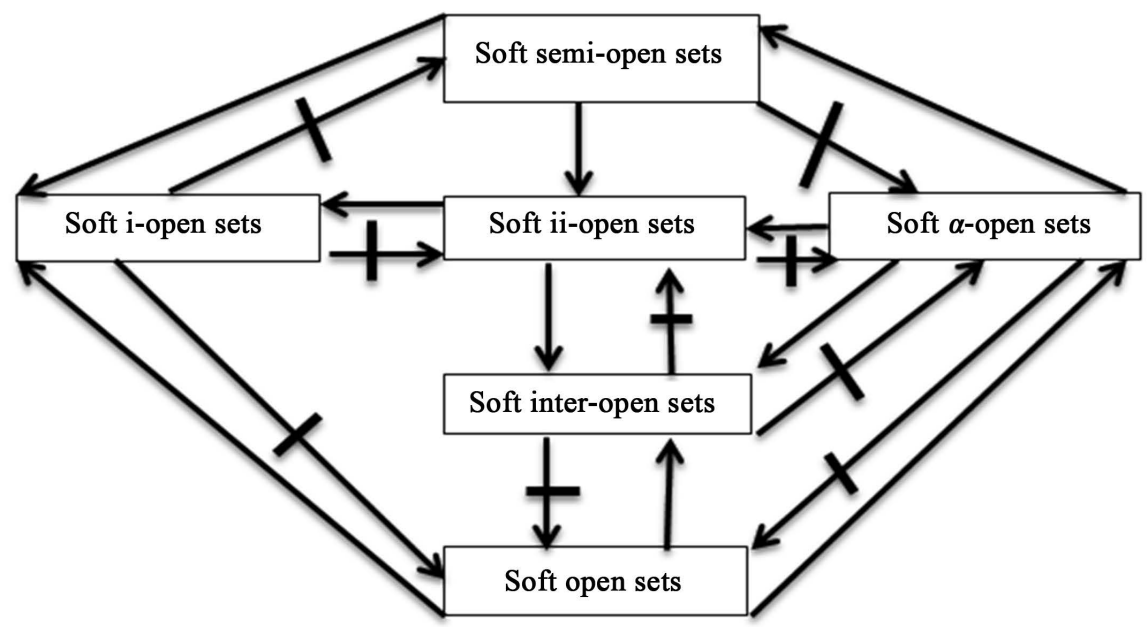

Figure 2. The relationship among soft ii-open sets and some other concepts of soft open sets.

\section{Some Applications of a Soft ii-Open Sets in the Soft Topological Spaces}

Definition 4.1: [3]. Consider $(K, E)$ as a soft set over $X$, and $A$ is a nonempty subdivision set of $X$. So, the sub-soft set of $(K, E)$ over $A$ indicated by $\left(A_{K}, E\right)$, has been known as $A_{K(e)}=A \cap K(e), \forall e \in E$. Specifically, $\left(A_{K}, E\right)=A \tilde{\bigcap}(K, E)$.

Definition 4.2: [3] [5]. Consider $(F, E)$ as soft set over $X$ and $x \in X$, consider $A$ be a nonempty subset of $X$. We define:

1) $(F, E) \backslash \tilde{\backslash}\{x\}$ as follows: $(F, E) \backslash \tilde{\{}\{x\}=\{F(e) \backslash\{x\}: \forall e \in E\}$.

2) $(F, E) \tilde{\cup} A$ as follows: $(F, E) \tilde{\cup} A=\{F(e) \cup A: \forall e \in E\}$.

3) $(F, E) \cong A$ if and only if $F(e)=A, \forall e \in E$.

4) $(F, E) \tilde{U}\{x\}$ as follows: $(F, E) \tilde{U}\{x\}=\{F(e) \cup\{x\}: \forall e \in E\}$.

5) $(F, E) \tilde{\bigcap}\{x\}$ as follows: $(F, E) \tilde{\cap}\{x\}=\{F(e) \cap\{x\}: \forall e \in E\}$.

6) Soft set $(F, E)$ containing a point $x \in X$, if $x \in F(e) \quad \forall e \in E$.

Definition 4.3: [22]. Consider $(G, E)$ as soft open set in $(X, \tau, E), e \in E$ and $x \in X$. It can be said that $(G, E)$ is e-soft open neighbor of $x$ whether $x \in G(e)$. "Further, a soft open set $(G, E)$ can be considered as a soft open neighbor of a point $x$ whether $x \in G(e), \forall e \in E$.

Definition 4.4: Consider $(F, E)$ be a soft set in $(X, \tau, E)$. A point $x \in X$ is said to be a limit point of $(F, E)$, if for each soft open neighborhood $(G, E)$ of $X$ we have $(F, E) \tilde{\cap}(G, E) \widetilde{\sim}\{x\} \neq \varnothing_{E}$. In other words A point $x \in X$ is said to be a limit point of $(F, E)$, if for each soft open set $(G, E)$ containing $x$ we have $(F, E) \tilde{\cap}(G, E) \backslash \tilde{\{}\left\{x \neq \varnothing_{E}\right.$. The set of all limit points of $(F, E)$ is called a derived set of $(F, E)$ and denoted by $D(F, E)$. A soft set $(F, E)$ is a soft closed if $D(F, E) \tilde{\sqsubseteq}(F, E)$ such that $D(F, E) \tilde{\sqsubseteq} F(e), \forall e \in E$.

Definition 4.5: A soft ii-open set $(G, E)$ in $(X, \tau, E)$ considered as soft ii-open neighbor of $x \in X$ if $x \in G(e) \quad \forall e \in E$.

Definition 4.6: Consider $(F, E)$ as a soft set in $(X, \tau, E)$. A point $x \in X$ is an ii-limit point of $(F, E)$ whether for every soft ii-open neighborhood $(G, E)$ of $X$ 
there is $(F, E) \tilde{\bigcap}(G, E) \tilde{\backslash}\{x\} \neq \varnothing_{E}$. In other words, a point $x \in X$ is an ii-limit point of $(F, E)$ whether every soft ii-open set $(G, E)$ which contain $x$ then there is $(F, E) \tilde{\bigcap}(G, E) \widetilde{\Upsilon}\{x\} \neq \varnothing_{E}$. The set of whole ii-limit points of $(F, E)$ known as ii-derived set of $(F, E)$ indicated by ii- $D(F, E)$. Note that a point $x \in X$ is not an ii-limit point of $(F, E)$ whether exist a soft ii-open set $(G, E)$ including $X$ where $(F, E) \tilde{\bigcap}(G, E) \backslash\{x\} \neq \varnothing_{E}$. A soft set $(F, E)$ is soft ii-closed if ii- $D(F, E) \tilde{\subseteq}(F, E)$ where ii- $D(F, E) \subseteq F(e), \forall e \in E$.

Theorem 4.7: Consider $(F, E),(B, E)$ as 2 soft sets in $(X, \tau, E)$, therefore, the next statements hold:

1) ii- $D(F, E) \subseteq D(F, E)$.

2) if $(F, E) \tilde{\subseteq}(B, E)$, then ii- $D(F, E) \subseteq \mathrm{ii}-D(B, E)$.

3) a) ii- $D(F, E) \bigcup \mathrm{ii}-D(B, E) \subseteq \mathrm{ii}-D((F, E) \tilde{U}(B, E))$.

b) $\operatorname{ii}-D((F, E) \tilde{\cap}(B, E)) \subseteq \mathrm{ii}-D(F, E) \cap \mathrm{ii}-D(B, E)$.

4) $\operatorname{ii-} D((F, E) \tilde{U}$ ii- $D(F, E)) \subseteq(F, E) \tilde{U}$ ii- $D(F, E)$.

\section{Proof:}

1) As each soft ii-open is a soft open set, it pursue that ii- $D(F, E) \subseteq D(F, E)$.

2) Consider $x \in \mathrm{ii}-D(F, E)$. Then there exist a soft ii-open set $(G, E)$ containing $x$ where $(F, E) \tilde{\cap}(G, E) \tilde{\backslash}\{x\} \neq \varnothing_{E} \quad(1)$. As $(F, E) \tilde{\subseteq}(B, E)$ we get $(F, E) \tilde{\cap}(B, E) \tilde{\subseteq}(B, E) \tilde{\cap}(G, E)$. It implies $(F, E) \tilde{\cap}(G, E) \backslash \tilde{}\{x\} \tilde{\subseteq}(B, E) \tilde{\bigcap}(G, E) \tilde{\backslash}\{x\}$, from (1) we have $(B, E) \tilde{\cap}(G, E) \tilde{\backslash}\{x\} \neq \varnothing_{E}$. Therefore, $x \in \mathrm{ii}-D(B, E)$. Hence ii- $D(F, E) \subseteq$ ii- $D(B, E)$.

3) a) Since $(F, E) \tilde{\subseteq}(F, E) \tilde{U}(B, E)$ and $(B, E) \tilde{\subseteq}(F, E) \tilde{U}(B, E)$, from (2) we have ii- $D(F, E) \subseteq$ ii- $D((F, E) \tilde{U}(B, E))$ and ii- $D(B, E) \subseteq$ ii- $D((F, E) \tilde{U}(B, E))$. This implies to ii- $D(F, E) \bigcup$ ii- $D(B, E) \subseteq$ ii- $D((F, E) \tilde{U}(B, E))$. b) Since $(F, E) \tilde{\cap}(B, E) \tilde{\subseteq}(F, E)$ and $(F, E) \tilde{\cap}(B, E) \tilde{\subseteq}(B, E)$, from (2) we get $\mathrm{ii}-D((F, E) \tilde{\cap}(B, E)) \subseteq \mathrm{ii}-D(F, E)$ and ii- $D((F, E) \tilde{\cap}(B, E)) \subseteq \mathrm{ii}-D(B, E)$. Hence ii- $D((F, E) \cap(B, E)) \subseteq \mathrm{ii}-D(F, E) \cap \mathrm{ii}-D(B, E)$.

4) Consider $x \in \mathrm{ii}-D((F, E) \tilde{U} \mathrm{ii}-D(F, E))$, so regarding a soft ii-open set $(G$, $E)$ containing $x$, we have $(G, E) \tilde{\bigcap}((F, E) \tilde{U}$ ii- $D(F, E)) \tilde{\backslash}\{x\} \neq \varnothing_{E}$. Thus $(G, E) \tilde{\cap}(F, E) \neq \varnothing_{E}$ or $(G, E) \tilde{\cap}(\mathrm{ii}-D(F, E) \tilde{\backslash}\{x\}) \neq \varnothing_{E}$. It follows that $(F, E) \backslash \tilde{\{}\left\{\tilde{\cap}(G, E) \neq \varnothing_{E}\right.$. Hence $x \in \mathrm{ii}-D(F, E)$ and in any case, ii- $D((F, E) \tilde{U}$ ii- $D(F, E)) \subseteq(F, E) \tilde{U}$ ii- $D(F, E)$

Theorem 4.8: For any soft set $(F, E)$ in $(X, \tau, E)$, ii- $C l(F, E)=(F, E) \tilde{U}$ ii- $D(F, E)$.

Proof: Since $(F, E) \tilde{\subseteq}$ ii- $C l(F, E)$ and by Theorem (4.7) (2) we have ii- $D(F, E) \tilde{\subseteq}$ ii- $D($ ii- $C l(F, E))$. Since ii- $C l(F, E)$ considered as soft ii-closed set we have ii- $D(\mathrm{ii}-C l(F, E)) \simeq$ ii- $C l(F, E)$, therefore,

$\mathrm{ii}-D(F, E) \tilde{\subseteq} \mathrm{ii}-C l(F, E)$. Hence $(F, E) \tilde{U} \mathrm{ii}-D(F, E) \tilde{\subseteq} \mathrm{ii}-C l(F, E)$. On the other hand, consider $x \tilde{\in}$ ii- $C l(F, E)$. If $x \tilde{\in}(F, E)$, this complete the evidence. If $x \tilde{\notin}(F, E)$, for each soft ii-open set $(G, E)$ that includes $x$ crossroads $(F, E)$ at a point distinct from $x$, so $x \in \mathrm{ii}-D(F, E)$. Thus,

ii- $C l(F, E) \tilde{\subseteq}(F, E) \tilde{U}$ ii- $D(F, E)$ 
Theorem 4.9: Consider $(F, E),(B, E)$ be two soft sets in $(X, \tau, E)$, so the next phrases hold:

1) ii- $\operatorname{Int}(F, E)$ is the union of all soft ii-open subsets of $(F, E)$.

2) $(F, E)$ is soft ii-open if and only if ii-Int $(F, E)=(F, E)$.

3) ii-Int (ii-Int $(F, E))=\mathrm{ii}-\operatorname{Int}(F, E)$.

4) ii-Int $(F, E)=(F, E) \widetilde{\text { ii }-D}\left(X_{E} \tilde{\Upsilon}(F, E)\right)$.

5) $X_{E} \tilde{\backslash} \mathrm{ii}-\operatorname{Int}(F, E)=\mathrm{ii}-C l\left(X_{E} \tilde{\backslash}(F, E)\right)=\mathrm{ii}-C l\left(F^{C}, E\right)$.

6) $X_{E} \widetilde{\Upsilon}$ ii- $C l(F, E)=\mathrm{ii}-\operatorname{Int}\left(X_{E} \tilde{\Upsilon}(F, E)\right)=\mathrm{ii}-\operatorname{Int}\left(F^{C}, E\right)$.

7) If $(F, E) \tilde{\subseteq}(B, E)$, then ii-Int $(F, E) \tilde{\subseteq}$ ii-Int $(B, E)$.

8) ii-Int $(F, E) \tilde{U} \mathrm{ii}-\operatorname{Int}(B, E) \tilde{\subseteq} \mathrm{ii}-\operatorname{Int}((F, E) \tilde{U}(B, E))$.

9) $\mathrm{ii}-\operatorname{Int}((F, E) \tilde{\cap}(B, E)) \tilde{\subseteq} \mathrm{ii}-\operatorname{Int}(F, E) \tilde{\bigcap} \mathrm{ii}-\operatorname{Int}(B, E)$.

\section{Proof:}

1) Consider $\left\{\left(G_{\mathrm{ii}}, E\right), \mathrm{ii} \in \Lambda\right\}$ as a group of whole soft ii-open subdivision sets of $(F, E)$. If $x \tilde{\in}$ ii-Int $(F, E)$, then there exist $j \in \Lambda$ where $x \tilde{\in}\left(G_{j}, E\right) \tilde{\subseteq}(F, E)$. Hence $x \tilde{\in} \tilde{\bigcup}_{\mathrm{ii} \in \Lambda}\left(G_{\mathrm{ii}}, E\right)$ and so ii-Int $(F, E) \tilde{\subseteq} \tilde{U}_{\mathrm{ii} \in \Lambda}\left(G_{\mathrm{ii}}, E\right)$. Contradict, if $y \tilde{\in} \tilde{U}_{\mathrm{ii} \in \Lambda}\left(G_{\mathrm{ii}}, E\right)$, then $y \tilde{\in}\left(G_{k}, E\right) \tilde{\subseteq}(F, E)$ for some $k \in \Lambda$. Thus $y \tilde{\in} \operatorname{ii-Int}(F, E)$ and $\tilde{U}_{\mathrm{ii} \in \Lambda}\left(G_{\mathrm{ii}}, E\right) \tilde{\subseteq} \operatorname{ii}-\operatorname{Int}(F, E)$.

2) Straight forward.

3) It pursues from (1) and (2).

4) If $x \tilde{\epsilon}(F, E) \tilde{\backslash} \mathrm{ii}-D\left(X_{E} \tilde{\backslash}(F, E)\right)$, then $x \tilde{\notin} \mathrm{ii}-D\left(X_{E} \tilde{\backslash}(F, E)\right)$, so there exist a soft ii-open set $(G, E)$ containing $x$ where $(G, E) \tilde{\bigcap}\left(X_{E} \widetilde{\Upsilon}(F, E)\right)=\phi_{E}$. Thus, $x \tilde{\in}(G, E) \tilde{\subseteq}(F, E)$ and hence $x \tilde{\in} \operatorname{ii-Int}(F, E)$. Thus shows $(F, E) \tilde{\backslash i \mathrm{ii}-D}\left(X_{E} \tilde{\backslash}(F, E)\right) \tilde{\subseteq} \mathrm{ii}-\operatorname{Int}(F, E)$. Now consider $x \tilde{\in} \mathrm{ii}-\operatorname{Int}(F, E)$. since ii-Int $(F, E)$ belongs to the group of whole soft open sets in $X$ and $\operatorname{ii}-\operatorname{Int}(F, E) \tilde{\cap}\left(X_{E} \tilde{\backslash}(F, E)\right)$, there is $x \notin \mathrm{ii}-D\left(X_{E} \tilde{\backslash}(F, E)\right)$. Therefore, ii-Int $(F, E)=(F, E) \tilde{\backslash i}$ i- $D\left(X_{E} \tilde{\backslash}(F, E)\right)$.

5) Using (4) and Theorem (4.8), we have,

$$
\begin{aligned}
& X_{E} \tilde{\backslash} \operatorname{ii}-I n t(F, E)=X_{E} \tilde{\backslash}\left((F, E) \tilde{\backslash i \mathrm{i}-D}\left(X_{E} \tilde{\backslash}(F, E)\right)\right) \\
& =\left(X_{E} \tilde{\backslash}(F, E)\right) \tilde{U} \operatorname{ii}-D\left(X_{E} \tilde{\backslash}(F, E)\right)=\mathrm{ii}-C l\left(X_{E} \tilde{\backslash}(F, E)\right)
\end{aligned} .
$$

6) Using (4) and Theorem (4.8) we get:

ii-Int $\left(X_{E} \tilde{\backslash}(F, E)\right)=\left(X_{E} \tilde{\backslash}(F, E)\right) \tilde{\mathrm{ii}}-D(F, E)$ $=\left(X_{E} \tilde{\backslash}(F, E)\right) \tilde{U} \mathrm{ii}-D(F, E)=X_{E} \tilde{\backslash} \mathrm{ii}-C l(F, E)$

7) Since $(F, E) \tilde{\subseteq}(B, E)$ and ii-Int $(F, E) \tilde{\subseteq}(F, E)$, ii-Int $(B, E) \tilde{\subseteq}(B, E)$, we get ii-Int $(F, E) \tilde{\subseteq}$ ii-Int $(B, E)$.

8) Since $(F, E) \tilde{\subseteq}((F, E) \tilde{U}(B, E))$ and $(B, E) \tilde{\subseteq}((F, E) \tilde{U}(B, E))$, from $(7)$ we get ii-Int $(F, E) \tilde{\subseteq}$ ii-Int $((F, E) \tilde{U}(B, E))$, ii-Int $(B, E) \tilde{\subseteq}$ ii-Int $((F, E) \tilde{U}(B, E))$. Therefore, ii-Int $(F, E) \tilde{U}$ ii-Int $(B, E) \tilde{\subseteq}$ ii-Int $((F, E) \tilde{U}(B, E))$.

9) Since $((F, E) \tilde{\cap}(B, E)) \tilde{\subseteq}(F, E)$ and $((F, E) \tilde{\cap}(B, E)) \tilde{\subseteq}(B, E)$, from (7) we get ii-Int $((F, E) \tilde{\cap}(B, E)) \tilde{\subseteq}$ ii-Int $(F, E)$ and 
ii-Int $((F, E) \tilde{\bigcap}(B, E)) \tilde{\subseteq}$ ii-Int $(B, E)$. Consequently, ii-Int $((F, E) \tilde{\cap}(B, E)) \tilde{\subseteq}$ ii-Int $(F, E) \tilde{\bigcap} \mathrm{ii}-\operatorname{Int}(B, E)$

Definition 4.10: Consider $(F, E)$ as soft set within $(X, \tau, E)$. We define the soft ii-border of $(F, E)$ indicated by ii-b $(F, E)$ as showed next:

ii- $b(F, E)=(F, E) \tilde{\backslash i}$ int $I(F, E)$.

Theorem 4.11: Consider $(F, E)$ as a soft set in $(X, \tau, E)$, therefore the next phrases holds:

1) ii- $b(F, E) \tilde{\subseteq} b(F, E)$ where $b(F, E)$ indicates the soft border of $(F, E)$ and defined as $b(F, E)=(F, E) \backslash \operatorname{Int}(F, E)$.

2) $\operatorname{ii}-\operatorname{Int}(F, E) \tilde{U} \mathrm{ii}-b(F, E)=(F, E)$.

3) $\mathrm{ii}-\operatorname{Int}(F, E) \tilde{\bigcap} \mathrm{ii}-b(F, E)=\phi_{E}$.

4) ii-b(F,E) $=\phi_{E}$ whether and only whether $(F, E)$ considered as soft ii-open set.

5) ii-b(ii-Int $(F, E))=\phi_{E}$.

6) $\mathrm{ii}-\operatorname{Int}(\mathrm{ii}-b(F, E))=\phi_{E}$.

7) ii- $b(\mathrm{ii}-b(F, E))=\mathrm{ii}-b(F, E)$.

8) $\mathrm{ii}-b(F, E)=(F, E) \tilde{\bigcap} \mathrm{ii}-C l\left(X_{E} \tilde{\Upsilon}(F, E)\right)$.

9) $\operatorname{ii}-b(F, E)=(F, E) \tilde{\bigcap}$ ii- $D\left(X_{E} \tilde{\backslash}(F, E)\right)$.

\section{Proof:}

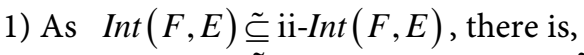

ii-b $(F, E)=(F, E) \widetilde{\sim}$ ii-Int $(F, E) \tilde{\subseteq}(F, E) \tilde{\backslash} \operatorname{Int}(F, E)=b(F, E)$.

2) Straightforward.

3) Straightforward.

4) Since ii-Int $(F, E) \tilde{\subseteq}(F, E)$, it follows based on the Theorem (4.9) (2), that $(F, E)$ can be a soft ii-open set whether and only whether $(F, E)=\operatorname{ii}-\operatorname{Int}(F, E)$ whether and only whether 1$)$ ii- $b(F, E)=(F, E) \backslash$ ii- $I n t(F, E)=\phi_{E}$.

5) Since ii-Int $(F, E)$ is a soft ii-open set following from (4) that ii- $b(\mathrm{ii}-\operatorname{Int}(F, E))=\phi_{E}$.

6) If $x \tilde{\in} \mathrm{ii}-\operatorname{Int}(\mathrm{ii}-b(F, E))$, then $x \tilde{\in} \mathrm{ii}-b(F, E)$. On the other hand, since $\mathrm{ii}-b(F, E) \tilde{\subseteq}(F, E), x \tilde{\in}$ ii-Int $(\mathrm{ii}-b(F, E)) \tilde{\subseteq} \mathrm{ii}-\operatorname{Int}(F, E)$. Hence, $x \tilde{\in}$ ii-Int $(F, E) \tilde{\bigcap} \mathrm{ii}-b(F, E)$. Which contradict with (3). Thus ii-Int $(\mathrm{ii}-b(F, E))=\phi_{E}$.

7) Using (6) we get ii- $b(\mathrm{ii}-b(F, E))=\mathrm{ii}-b(F, E) \widetilde{\text { ii }-I n t}(\mathrm{ii}-b(F, E))=\mathrm{ii}-b(F, E)$.

8) Using Theorem (4.9) (6), we have $\mathrm{ii}-b(F, E)=(F, E) \tilde{\mathrm{ii}}-\operatorname{Int}(F, E)=(F, E) \tilde{\backslash}(X \tilde{\backslash \mathrm{ii}-C l}(X(F, E)))$ $=(F, E) \tilde{\bigcap} \mathrm{ii}-C l(X \tilde{\backslash}(F, E))$

9) Applying (8) and Theorem (4.8), we have ii- $b(F, E)=(F, E) \tilde{\cap} \mathrm{ii}-C l(X \tilde{\backslash}(F, E))$ $=(F, E) \tilde{\cap}(X \tilde{\urcorner}(F, E)) \tilde{\mathrm{Uii}}-D(X \tilde{\backslash}(F, E))=(F, E) \tilde{\cap} \mathrm{ii}-D(X \tilde{\backslash}(F, E)) \cdot$

Definition 4.12: Consider $(F, E)$ as a soft open in $(X, \tau, E)$. We define the soft 
ii-Frontier of $(F, E)$ indicated thru ii- $F r(F, E)$ as pursues: $\mathrm{ii}-F r(F, E)=\mathrm{ii}-C l(F, E) \backslash \tilde{\mathrm{ii}}-\operatorname{Int}(F, E)$.

Theorem 4.13: Consider $(F, E)$ as a soft set in $(X, \tau, E)$, so the next statements hold:

1) ii- $F r(F, E) \tilde{\subseteq} \operatorname{Fr}(F, E)$ where $\operatorname{Fr}(F, E)$ denotes the soft frontier of $(F, E)$ and known as $\operatorname{Fr}(F, E)=C l(F, E) \tilde{\operatorname{Int}}(F, E)$.

2) $\mathrm{ii}-C l(F, E)=\mathrm{ii}-\operatorname{Int}(F, E) \tilde{U} \mathrm{ii}-F r(F, E)$.

3) ii-Int $(F, E) \tilde{\bigcap} \mathrm{ii}-F r(F, E)=\phi_{E}$.

4) ii-b( $F, E) \tilde{\subseteq}$ ii- $F r(F, E)$.

5) $\mathrm{ii}-F r(F, E)=\mathrm{ii}-b(F, E) \tilde{U} \mathrm{ii}-D(F, E)$.

6) $\mathrm{ii}-F r(F, E) \cong \mathrm{ii}-D(F, E)$ whether and only whether $(F, E)$ considered as a soft ii-open.

7) $\mathrm{ii}-F r(F, E)=\mathrm{ii}-C l(F, E) \tilde{\bigcap} \mathrm{ii}-C l\left(X_{E} \tilde{\backslash}(F, E)\right)$.

8) $\mathrm{ii}-F r(F, E)=\mathrm{ii}-F r\left(X_{E} \sim(F, E)\right)$.

9) ii- $F r(F, E)$ is soft ii-closed set.

10) ii- $F r(\mathrm{ii}-F r(F, E)) \tilde{\subseteq} \mathrm{ii}-\operatorname{Fr}(F, E)$.

11) $\mathrm{ii}-\operatorname{Fr}(\mathrm{ii}-\operatorname{Int}(F, E)) \simeq \mathrm{ii}-\operatorname{Fr}(F, E)$.

12) ii- $F r(\mathrm{ii}-C l(F, E)) \subseteq$ ii- $F r(F, E)$.

13) ii-Int $(F, E)=(F, E) \backslash$ ii- $F r(F, E)$.

\section{Proof:}

1) Since ii- $C l(F, E) \tilde{\subseteq} C l(F, E)$ and $\operatorname{Int}(F, E) \tilde{\subseteq}$ ii-Int $(F, E)$, it follows that: $\mathrm{ii}-F r(F, E)=\mathrm{ii}-C l(F, E) \tilde{\backslash i \mathrm{i}-I n t}(F, E) \tilde{\subseteq} C l(F, E) \sim \tilde{I n t}(F, E)=\operatorname{Fr}(F, E)$.

2) $\mathrm{ii}-\operatorname{Int}(F, E) \tilde{U} \mathrm{ii}-F r(F, E)=\mathrm{ii}-\operatorname{Int}(F, E) \tilde{U}(\mathrm{ii}-C l(F, E) \widetilde{i i}-\operatorname{Int}(F, E))$ $=\mathrm{ii}-C l(F, E)$

3) $\operatorname{ii-Int}(F, E) \tilde{\bigcap} \mathrm{ii}-F r(F, E)=\mathrm{ii}-\operatorname{Int}(F, E) \tilde{\bigcap}(\mathrm{ii}-C l(F, E) \tilde{\text { ii- } I n t}(F, E))=\phi_{E}$.

4) Since $(F, E) \tilde{\subseteq} \mathrm{ii}-C l(F, E)$, we have

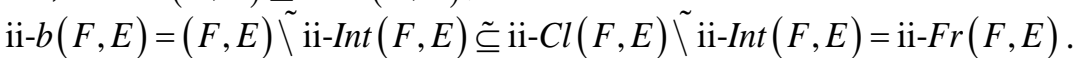

5) Since ii-Int $(F, E) \tilde{U}$ ii- $F r(F, E)=\operatorname{ii}-\operatorname{Int}(F, E) \tilde{U}$ ii- $b(F, E) \tilde{U}$ ii- $D(F, E)$, we have ii- $F r(F, E)=$ ii- $b(F, E) \tilde{U}$ ii- $D(F, E)$.

6) Assume $(F, E)$ considered as soft ii-open. Then utilizing Theorem (4.9) (2) and Theorem (4.11) (4) (9), we have,

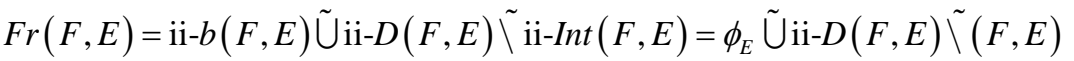
$=\mathrm{ii}-D(F, E) \widetilde{(}(F, E) \cong \mathrm{ii}-b\left(X_{E} \tilde{\backslash}(F, E)\right)$

Conversely, suppose that $\mathrm{ii}-F r(F, E)=\mathrm{ii}-b\left(X_{E} \tilde{\backslash}(F, E)\right)$. Then $\phi_{E}=\mathrm{ii}-F r(F, E) \widetilde{i i}-b\left(X_{E} \tilde{\nearrow}(F, E)\right)$

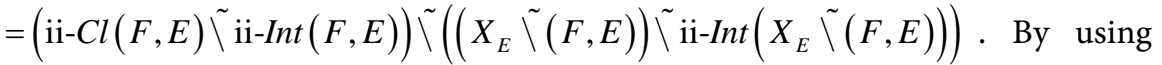
$=(F, E) \tilde{\text { ii }-I n t}(F, E)$

(4) and (5) of Theorem (4.9) and so $(F, E) \tilde{\subseteq}$ ii-Int $(F, E)$. Since ii-Int $(F, E) \tilde{\subseteq}(F, E)$ in general, they pursue that ii-Int $(F, E)=(F, E)$. So from Theorem (4.9) (2) $(F, E)$ is considered to be soft ii-open set. 


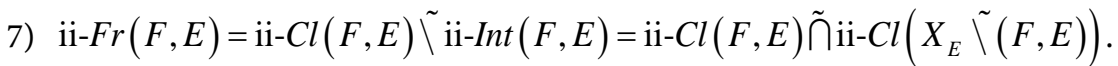

8) It follows from (7).

$$
\begin{aligned}
& \mathrm{ii}-C l(\mathrm{ii}-F r(F, E))=\mathrm{ii}-C l\left(\mathrm{ii}-C l(F, E) \tilde{\cap} \mathrm{ii}-C l\left(X_{E}(F, E)\right)\right) \\
9) & \tilde{\subseteq} \mathrm{ii}-C l(\mathrm{ii}-C l(F, E)) \tilde{\cap} \mathrm{ii}-C l\left(\mathrm{ii}-C l\left(X_{E} \tilde{\backslash}(F, E)\right)\right) \\
& =\mathrm{ii}-C l(F, E) \backslash \tilde{\mathrm{ii}}-\operatorname{Int}(F, E)=\mathrm{ii}-F r(F, E)
\end{aligned}
$$

$E)$ is soft ii-closed.

10)

$$
\begin{aligned}
& \mathrm{ii}-F r(\mathrm{ii}-F r(F, E))=\mathrm{ii}-C l(\mathrm{ii}-F r(F, E)) \tilde{\cap} \mathrm{ii}-C l\left(X_{E} \tilde{\backslash} \tilde{\mathrm{ii}}-F r(F, E)\right) \\
& \tilde{\subseteq} \mathrm{ii}-C l(\mathrm{ii}-F r(F, E))=\mathrm{ii}-F r(F, E)
\end{aligned}
$$

11) Using Theorem (3.2.9) (3), we get

ii- $F r(\operatorname{ii}-\operatorname{Int}(F, E))=$ ii- $C l($ ii-Int $(F, E)) \sim$ ii-Int $(\operatorname{ii}-\operatorname{Int}(F, E))$

$\tilde{\subseteq} \mathrm{ii}-C l(F, E) \tilde{\sim} \mathrm{ii}-\operatorname{Int}(F, E)=\mathrm{ii}-F r(F, E)$

12) Since $(F, E) \tilde{\subseteq} \mathrm{ii}-C l(F, E)$, we have

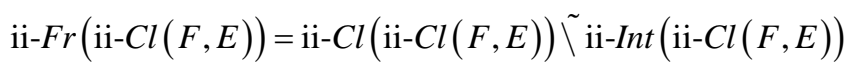$$
=\mathrm{ii}-C l(F, E) \tilde{\text { ii }-I n t}(\mathrm{ii}-C l(F, E)) \tilde{\subseteq} \mathrm{ii}-C l(F, E) \tilde{\mathrm{ii}-} \operatorname{Int}(F, E)=\mathrm{ii}-F r(F, E) \cdot
$$

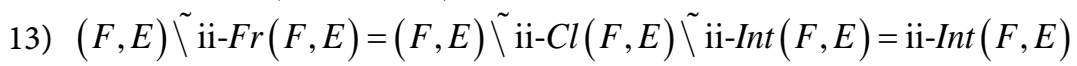

Definition 4.14: Consider $(F, E)$ as soft set within $(X, \tau, E)$. We define the soft ii-Exterior of $(F, E)$ indicated by ii-Ext $(F, E)$ as:

ii-Ext $(F, E)=\operatorname{ii}-\operatorname{Int}\left(X_{E} \tilde{\Upsilon}(F, E)\right)$.

Theorem 4.15: Consider $(F, E)$ as a soft set within $(X, \tau, E)$, so next phrases means:

1) $\operatorname{Ext}(F, E) \tilde{\subseteq} \mathrm{ii}-\operatorname{Ext}(F, E)$ where $\operatorname{Ext}(F, E)$ denotes the soft exterior of $(F$, $E)$ and known as $\operatorname{Ext}(F, E)=\operatorname{Int}\left(X_{E} \Upsilon^{\tilde{}}(F, E)\right)$.

2) ii-Ext $(F, E)$ is soft ii-open set.

3) $\mathrm{ii}-\operatorname{Ext}(F, E)=\mathrm{ii}-\operatorname{Int}\left(X_{E} \tilde{\backslash}(F, E)\right)=X_{E} \tilde{\backslash} \mathrm{ii}-C l(F, E)$.

4) ii-Ext(ii-Ext $(F, E))=$ ii-Int $(\mathrm{ii}-C l(F, E))$.

5) If $\left(F_{1}, E\right) \tilde{\subseteq}\left(F_{2}, E\right)$, then ii-Ext $\left(F_{2}, E\right) \tilde{\subseteq}$ ii-Ext $\left(F_{1}, E\right)$.

6) ii-Ext $\left(\left(F_{1}, E\right) \tilde{U}\left(F_{2}, E\right)\right) \tilde{\subseteq}$ ii-Ext $\left(F_{1}, E\right) \tilde{U}$ ii-Ext $\left(F_{2}, E\right)$.

7) ii-Ext $\left(F_{1}, E\right) \tilde{\cap} \mathrm{ii}-\operatorname{Ext}\left(F_{2}, E\right) \tilde{\subseteq} \mathrm{ii}-\operatorname{Ext}\left(\left(F_{1}, E\right) \tilde{\cap}\left(F_{2}, E\right)\right)$.

8) $\mathrm{ii}-\operatorname{Ext}\left(X_{E}\right)=\phi_{E}$.

9) $\operatorname{ii}-\operatorname{Ext}\left(\phi_{E}\right)=X_{E}$.

10) $\mathrm{ii}-\operatorname{Ext}(F, E)=\mathrm{ii}-\operatorname{Ext}\left(X_{E} \tilde{\backslash} \mathrm{ii}-\operatorname{Ext}(F, E)\right)$.

11) ii-Int $(F, E) \tilde{\subseteq}$ ii-Ext $(\mathrm{ii}-\operatorname{Ext}(F, E))$.

12) $X_{E}=\mathrm{ii}-\operatorname{Int}(F, E) \tilde{U} \mathrm{ii}-\operatorname{Ext}(F, E) \tilde{U} \mathrm{ii}-F r(F, E)$.

\section{Proof:}

1) As $\operatorname{Int}(F, E) \tilde{\subseteq} \mathrm{ii}-\operatorname{Int}(F, E)$, we have,

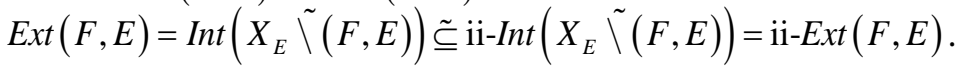

2) It follows from Theorem (4.9) (1).

3) It is straightforward by Theorem (4.9) (6).

4)

$$
\begin{aligned}
& \text { ii-Ext }(\mathrm{ii}-\operatorname{Ext}(F, E))=\mathrm{ii}-\operatorname{Ext}\left(X_{E} \tilde{\backslash} \mathrm{ii}-C l(F, E)\right) \\
& =\mathrm{ii}-\operatorname{Int}\left(X_{E} \tilde{\backslash} X_{E} \tilde{\backslash} \mathrm{ii}-C l(F, E)\right)=\mathrm{ii}-\operatorname{Int}(\mathrm{ii}-C l(F, E))
\end{aligned}
$$


5) Assume that $\left(F_{1}, E\right) \tilde{\subseteq}\left(F_{2}, E\right)$, then utilizing Theorem (4.9) (7), we have ii-Ext $\left(F_{2}, E\right)=\operatorname{ii}-I n t\left(X_{E} \tilde{\nearrow}\left(F_{2}, E\right)\right) \tilde{\subseteq} \operatorname{ii}-\operatorname{Int}\left(X_{E} \tilde{\nearrow}\left(F_{1}, E\right)\right)=\operatorname{ii}-\operatorname{Ext}\left(F_{1}, E\right)$.

6) Applying Theorem (4.9) (8), we get

$$
\begin{aligned}
& \text { ii-Ext }\left(\left(F_{1}, E\right) \tilde{U}\left(F_{2}, E\right)\right)=\operatorname{ii-Int}\left(X_{E}\left(\left(F_{1}, E\right) \tilde{U}\left(F_{2}, E\right)\right)\right) \\
& \tilde{\subseteq} \operatorname{ii}-\operatorname{Int}\left(X_{E} \tilde{\backslash}\left(F_{1}, E\right)\right) \tilde{U} \operatorname{ii}-\operatorname{Int}\left(X_{E} \tilde{\Upsilon}\left(F_{2}, E\right)\right) \\
& =\mathrm{ii}-\operatorname{Ext}\left(F_{1}, E\right) \tilde{U} \mathrm{ii}-\operatorname{Ext}\left(F_{2}, E\right)
\end{aligned}
$$

7) Applying Theorem (4.9) (9), we obtain

$\mathrm{ii}-\operatorname{Ext}\left(F_{1}, E\right) \tilde{\bigcap} \mathrm{ii}-\operatorname{Ext}\left(F_{2}, E\right)=\mathrm{ii}-\operatorname{Int}\left(X_{E} \tilde{\backslash}\left(F_{1}, E\right)\right) \tilde{\cap} \mathrm{ii}-\operatorname{Int}\left(X_{E} \tilde{\backslash}\left(F_{2}, E\right)\right)$

$\tilde{\subseteq} \mathrm{ii}-I n t\left(\left(X_{E} \tilde{\backslash}\left(F_{1}, E\right)\right) \tilde{\subseteq}\left(X_{E} \widetilde{\Upsilon}\left(F_{2}, E\right)\right)\right)=\mathrm{ii}-\operatorname{Int}\left(X_{E} \widetilde{\nearrow}\left(\left(F_{1}, E\right) \tilde{\cap}\left(F_{2}, E\right)\right)\right)$.

$=\mathrm{ii}-\operatorname{Ext}\left(\left(F_{1}, E\right) \tilde{\bigcap}\left(F_{2}, E\right)\right)$

8) Straightforward.

9) Straightforward.

$$
\begin{aligned}
& \operatorname{ii}-\operatorname{Ext}\left(X_{E} \tilde{\backslash} \operatorname{ii}-\operatorname{Ext}(F, E)\right)=\operatorname{ii}-\operatorname{Ext}\left(X_{E} \tilde{\backslash} \operatorname{ii}-\operatorname{Int}\left(X_{E} \tilde{\backslash}(F, E)\right)\right) \\
& \text { 10) }=\mathrm{ii}-\operatorname{Int}\left(X_{E} \tilde{\backslash}\left(X_{E} \tilde{\backslash} \mathrm{ii}-\operatorname{Int}\left(X_{E} \tilde{\backslash}(F, E)\right)\right)\right)=\mathrm{ii}-\operatorname{Int}\left(\mathrm{ii}-\operatorname{Int}\left(X_{E} \tilde{\backslash}(F, E)\right)\right) \text {. } \\
& =\mathrm{ii}-\operatorname{Int}\left(X_{E} \tilde{\backslash}(F, E)\right)=\mathrm{ii}-\operatorname{Ext}(F, E) \\
& \text { 11) } \\
& \mathrm{ii}-\operatorname{Int}(F, E) \tilde{\subseteq} \mathrm{ii}-\operatorname{Int}(\mathrm{ii}-C l(F, E))=\mathrm{ii}-\operatorname{Int}\left(X_{E} \tilde{\backslash} \mathrm{ii}-\operatorname{Int}\left(X_{E} \widetilde{\nearrow}(F, E)\right)\right) \\
& =\mathrm{ii}-\operatorname{Int}\left(X_{E} \tilde{\Upsilon} \mathrm{ii}-\operatorname{Ext}(F, E)\right)=\mathrm{ii}-\operatorname{Ext}(\mathrm{ii}-\operatorname{Ext}(F, E)) \\
& \text { ii-Int }(F, E) \tilde{U} \mathrm{ii}-\operatorname{Ext}(F, E) \tilde{U} \mathrm{ii}-F r(F, E) \\
& \text { 12) }=\mathrm{ii}-\operatorname{Int}(F, E) \tilde{U} \mathrm{ii}-\operatorname{Int}\left(X_{E} \tilde{\backslash}(F, E)\right) \tilde{U}(\mathrm{ii}-C l(F, E) \tilde{\operatorname{ii}-I n t}(F, E)) \text {. } \\
& =\operatorname{ii}-\operatorname{Int}(F, E) \tilde{U}\left(X_{E} \tilde{\backslash} \mathrm{ii}-C l(F, E)\right) \tilde{U}(\mathrm{ii}-C l(F, E) \tilde{\operatorname{ii}-I n t}(F, E))=X_{E}
\end{aligned}
$$

Example 4.16: Consider $X=\left\{x_{1}, x_{2}, x_{3}\right\}$,

$\tau=\left\{\phi_{E},\left(F_{1}, E\right),\left(F_{2}, E\right),\left(F_{3}, E\right), X_{E}\right\}, \quad E=\left\{e_{1}, e_{2}\right\}$. Where

$\left(F_{1}, E\right)=\left\{\left(e_{1},\left\{x_{1}\right\}\right),\left(e_{2},\left\{x_{1}\right\}\right)\right\},\left(F_{2}, E\right)=\left\{\left(e_{1},\left\{x_{2}\right\}\right),\left(e_{2},\left\{x_{2}\right\}\right)\right\}$,

$\left(F_{3}, E\right)=\left\{\left(e_{1},\left\{x_{1}, x_{2}\right\}\right),\left(e_{2},\left\{x_{1}, x_{2}\right\}\right)\right\}$.

$O S\left(X_{E}\right)=\left\{\phi_{E},\left(F_{1}, E\right),\left(F_{2}, E\right),\left(F_{3}, E\right), X\right\}$,

$\operatorname{CS}\left(X_{E}\right)=\left\{X,\left(F_{1}, E\right)^{c}=\left(F_{4}, E\right)=\left\{\left(e_{1},\left\{x_{2}, x_{3}\right\}\right),\left(e_{2},\left\{x_{2}, x_{3}\right\}\right)\right\}\right.$,

$$
\begin{aligned}
& \left(F_{2}, E\right)^{c}=\left(F_{5}, E\right)=\left\{\left(e_{1},\left\{x_{1}, x_{3}\right\}\right),\left(e_{2},\left\{x_{1}, x_{3}\right\}\right)\right\}, . \\
& \left.\left(F_{3}, E\right)^{c}=\left(F_{6}, E\right)=\left\{\left(e_{1},\left\{x_{3}\right\}\right),\left(e_{2},\left\{x_{3}\right\}\right)\right\}, \phi_{E}\right\}
\end{aligned}
$$

Soft i-open sets are: $\phi_{E},\left(F_{1}, E\right),\left(F_{2}, E\right),\left(F_{3}, E\right),\left(F_{4}, E\right),\left(F_{5}, E\right), X$.

Soft ii-open sets are: $\phi_{E},\left(F_{1}, E\right),\left(F_{2}, E\right),\left(F_{3}, E\right),\left(F_{4}, E\right),\left(F_{5}, E\right), X$.

Soft ii-closed sets are: $\phi_{E},\left(F_{1}, E\right),\left(F_{2}, E\right),\left(F_{4}, E\right),\left(F_{5}, E\right),\left(F_{6}, E\right), X$.

Consider $\left(F_{L}, E\right)=\left\{\left(e_{1},\left\{x_{1}, x_{3}\right\}\right),\left(e_{2},\left\{x_{1}, x_{3}\right\}\right)\right\}$,

1) ii- $D\left(F_{L}, E\right)=\varnothing \tilde{\subseteq}\left(F_{L}, E\right)$. Obviously $\left(F_{L}, E\right)$ is considered as soft

ii-closed set. Consider $\left(F_{K}, E\right)=\left\{\left(e_{1},\left\{x_{1}, x_{2}\right\}\right),\left(e_{2},\left\{x_{1}, x_{2}\right\}\right)\right\}$.

2) ii- $D\left(F_{K}, E\right)=\left\{x_{3}\right\} \tilde{\Psi}\left(F_{K}, E\right)$. Clearly $\left(F_{K}, E\right)$ is not soft ii-closed set.

3) $\operatorname{ii}-D\left(F_{K}, E\right)=\left\{x_{3}\right\} \simeq D\left(F_{K}, E\right)$. 
4) $\operatorname{ii}-C l\left(F_{K}, E\right)=X_{E}=\left(F_{K}, E\right) \tilde{U}$ ii- $D\left(F_{K}, E\right)$.

5) ii-Int $\left(F_{K}, E\right)=X_{E}=\left(F_{1}, E\right) \tilde{U}\left(F_{2}, E\right) \tilde{U} \phi_{E}=\left(F_{3}, E\right)=\left(F_{K}, E\right)$. Clearly $\left(F_{K}, E\right)$ is soft ii-open set.

6) ii-Int $\left(\mathrm{ii}-\operatorname{Int}\left(F_{K}, E\right)\right)=\left(F_{K}, E\right)=\mathrm{ii}-\operatorname{Int}\left(F_{K}, E\right)$.

7) $\operatorname{ii}-D\left(F_{6}, E\right)=\varnothing$,

$$
\left(F_{K}, E\right) \widetilde{i i}-D\left(X_{E} \tilde{\backslash}(F, E)\right)=\left(F_{K}, E\right) \tilde{i i}-D\left(F_{6}, E\right)
$$

$$
=\left(F_{K}, E\right) \tilde{\backslash} \varnothing=\left(F_{K}, E\right)=\mathrm{ii}-\operatorname{Int}\left(F_{K}, E\right)
$$

8) $\operatorname{ii}-C l\left(X_{E} \tilde{\backslash}\left(F_{K}, E\right)\right)=\operatorname{ii}-C l\left(F_{6}, E\right)=\left(F_{6}, E\right)=X_{E} \tilde{\Upsilon} \operatorname{ii}-I n t\left(F_{k}, E\right)$.

9) $X_{E} \tilde{\Upsilon} \mathrm{ii}-C l\left(F_{k}, E\right)=X_{E} \tilde{\Upsilon} X_{E}=\varnothing_{E}=\operatorname{ii}-I n t\left(X_{E} \tilde{\backslash}\left(F_{K}, E\right)\right)$.

10) $\left(F_{6}, E\right) \tilde{\subseteq}\left(F_{L}, E\right)$, ii-Int $\left(F_{6}, E\right)=\varnothing_{E} \subset\left(F_{5}, E\right)=\operatorname{ii}-\operatorname{Int}\left(F_{L}, E\right)$.

11) ii-Int $\left(F_{6}, E\right) \tilde{U} \operatorname{ii}-\operatorname{Int}\left(F_{L}, E\right)=\left(F_{5}, E\right)=\mathrm{ii}-\operatorname{Int}\left(\left(F_{6}, E\right) \tilde{U}\left(F_{L}, E\right)\right)$.

12) ii-Int $\left(\left(F_{6}, E\right) \tilde{\cap}\left(F_{L}, E\right)\right)=\varnothing_{E}=\mathrm{ii}-\operatorname{Int}\left(F_{6}, E\right) \tilde{\cap} \mathrm{ii}-\operatorname{Int}\left(F_{L}, E\right)$.

13) ii-b $\left(F_{K}, E\right)=\left(F_{K}, E\right) \widetilde{i i}-\operatorname{Int}\left(F_{K}, E\right)=\left(F_{K}, E\right) \tilde{\backslash}\left(F_{K}, E\right)=\varnothing_{E}$,

$b\left(F_{K}, E\right)=\left(F_{K}, E\right) \tilde{\backslash} \operatorname{Int}\left(F_{K}, E\right)=\left(F_{K}, E\right) \backslash\left(F_{K}, E\right)=\varnothing_{E}$. Hence

ii-b( $\left.F_{K}, E\right) \tilde{\subseteq} b\left(F_{K}, E\right)$.

14) $\operatorname{ii-Int}\left(F_{K}, E\right) \tilde{U} \mathrm{ii}-b\left(F_{K}, E\right)=\left(F_{K}, E\right) \tilde{U} \varnothing_{E}=\left(F_{K}, E\right)$.

15) ii-Int $\left(F_{K}, E\right) \tilde{\bigcap} \mathrm{ii}-b\left(F_{K}, E\right)=\left(F_{K}, E\right) \tilde{\cap} \varnothing_{E}=\varnothing_{E}$.

16) ii-b $\left(F_{K}, E\right)=\varnothing_{E}$, hence $\left(F_{K}, E\right)$ is soft ii-open set, ii- $b\left(F_{L}, E\right)=\varnothing_{E}$, hence $\left(F_{L}, E\right)$ is soft ii-open set, ii- $b\left(F_{6}, E\right)=\left(F_{6}, E\right)$, hence $\left(F_{\odot} E\right)$ is not soft ii-open set.

17) $\mathrm{ii}-b\left(\mathrm{ii}-\operatorname{Int}\left(F_{K}, E\right)\right)=\mathrm{ii}-b\left(F_{K}, E\right)=\varnothing_{E}$, ii-Int $\left(\right.$ ii $\left.-b\left(F_{K}, E\right)\right)=$ ii-Int $\left(\varnothing_{E}\right)=\varnothing_{E}$. ii- $b\left(\mathrm{ii}-b\left(F_{6}, E\right)\right)=\mathrm{ii}-b\left(F_{6}, E\right)=\left(F_{6}, E\right)$.

18) $\operatorname{ii}-C l\left(X_{E} \Upsilon \widetilde{\Upsilon}\left(F_{6}, E\right)\right)=\operatorname{ii}-C l\left(F_{3}, E\right)=X_{E}$, $\left(F_{6}, E\right) \tilde{\bigcap} \mathrm{ii}-C l\left(X_{E} \tilde{\Upsilon}\left(F_{6}, E\right)\right)=\left(F_{6}, E\right) \tilde{\cap} X_{E}=\left(F_{6}, E\right)=\mathrm{ii}-b\left(F_{6}, E\right)$.

19) $\left(F_{6}, E\right) \tilde{\cap} \mathrm{ii}-D\left(X_{E} \tilde{\Upsilon}\left(F_{6}, E\right)\right)=\left(F_{6}, E\right) \tilde{\cap} \mathrm{ii}-D\left(F_{K}, E\right)=\left(F_{6}, E\right) \tilde{\cap}\left\{x_{3}\right\}=\left(F_{6}, E\right)$, hence ii-b $\left(F_{6}, E\right)=\left(F_{6}, E\right) \tilde{\bigcap} \mathrm{ii}-D\left(X_{E} \tilde{\Upsilon}\left(F_{6}, E\right)\right)$.

20) ii-Fr $\left(F_{6}, E\right)=\mathrm{ii}-C l\left(F_{6}, E\right) \tilde{\text { ii }-I n t}\left(F_{6}, E\right)=\left(F_{6}, E\right) \tilde{\varnothing}_{E}=\left(F_{6}, E\right)$, $\operatorname{Fr}\left(F_{6}, E\right)=\operatorname{Cl}\left(F_{6}, E\right) \tilde{\operatorname{Int}}\left(F_{6}, E\right)=\left(F_{6}, E\right) \backslash \varnothing_{E}=\left(F_{6}, E\right)$. Hence ii- $F r\left(F_{6}, E\right) \tilde{\subseteq} \operatorname{Fr}\left(F_{6}, E\right)$.

21) ii-Cl $\left(F_{6}, E\right)=\left(F_{6}, E\right)=\mathrm{ii}-\operatorname{Int}\left(F_{6}, E\right) \tilde{U} \mathrm{ii}-F r\left(F_{6}, E\right)$, ii- $b\left(F_{6}, E\right)=\left(F_{6}, E\right) \tilde{\subseteq}$ ii- $F r\left(F_{6}, E\right)$, ii- $D\left(F_{6}, E\right)=\varnothing \neq \mathrm{ii}-F r\left(F_{6}, E\right),\left(F_{\sigma}, E\right)$ is not soft ii-open set, ii- $C l\left(X_{E} \tilde{\Upsilon}\left(F_{6}, E\right)\right)=\mathrm{ii}-C l\left(F_{K}, E\right)=X_{E}$,

$\mathrm{ii}-\operatorname{Fr}\left(F_{6}, E\right)=\mathrm{ii}-C l\left(F_{6}, E\right) \cap \tilde{\cap i}-C l\left(X_{E} \backslash\left(F_{6}, E\right)\right)$, $\mathrm{ii}-\operatorname{Fr}\left(X_{E} \tilde{\Upsilon}\left(F_{6}, E\right)\right)=\mathrm{ii}-F r\left(F_{K}, E\right)=\left(F_{6}, E\right)=\mathrm{ii}-F r\left(F_{6}, E\right)$, ii- $F r\left(F_{6}, E\right)=\left(F_{6}, E\right),\left(F_{\odot} E\right)$ is soft ii-closed set, ii- $\operatorname{Fr}\left(\mathrm{ii}-\operatorname{Fr}\left(F_{6}, E\right)\right)=\mathrm{ii}-\operatorname{Fr}\left(F_{6}, E\right)=\left(F_{6}, E\right)$, ii- $F r\left(\mathrm{ii}-C l\left(F_{6}, E\right)\right)=\mathrm{ii}-\operatorname{Fr}\left(F_{6}, E\right)=\left(F_{6}, E\right) \tilde{\subseteq} \mathrm{ii}-F r\left(F_{6}, E\right)$, $\left(F_{6}, E\right) \tilde{\backslash i \mathrm{ii}-F r}\left(F_{6}, E\right)=\left(F_{6}, E\right) \tilde{\backslash}\left(F_{6}, E\right)=\varnothing_{E}=\operatorname{ii}-\operatorname{Int}\left(F_{6}, E\right)$.

22) ii-Ext $\left(F_{K}, E\right)=\mathrm{ii}-\operatorname{Int}\left(X_{E} \tilde{\backslash}\left(F_{K}, E\right)\right)=\mathrm{ii}-\operatorname{Int}\left(F_{6}, E\right)=\varnothing_{E}$, $\operatorname{Ext}\left(F_{K}, E\right)=\operatorname{Int}\left(X_{E} \tilde{\backslash}\left(F_{K}, E\right)\right)=\operatorname{Int}\left(F_{6}, E\right)=\varnothing_{E} \tilde{\subseteq} \mathrm{ii}-\operatorname{Ext}\left(F_{K}, E\right)$, ii-Ext $\left(F_{L}, E\right)=\mathrm{ii}-\operatorname{Int}\left(X_{E} \widetilde{\Upsilon}\left(F_{L}, E\right)\right)=\operatorname{ii}-\operatorname{Int}\left(F_{2}, E\right)=\left(F_{2}, E\right)$ is soft ii-open set, $X_{E} \tilde{\backslash} \mathrm{ii}-C l\left(F_{L}, E\right)=X_{E} \tilde{\Upsilon}\left(F_{5}, E\right)=\left(F_{2}, E\right)=\mathrm{ii}-E x t\left(F_{L}, E\right)=\operatorname{ii}-\operatorname{Int}\left(X_{E}\left(F_{L}, E\right)\right)$, 


$$
\begin{aligned}
& \mathrm{ii}-\operatorname{Ext}\left(\mathrm{ii}-\operatorname{Ext}\left(F_{L}, E\right)\right)=\mathrm{ii}-\operatorname{Ext}\left(F_{2}, E\right)=\mathrm{ii}-\operatorname{Int}\left(X_{E} \widetilde{\backslash}\left(F_{2}, E\right)\right)=\mathrm{ii}-\operatorname{Int}\left(F_{5}, E\right) \\
& =\left(F_{5}, E\right)=\left(F_{L}, E\right) \\
& \text { ii-Int }\left(\text { ii- } C l\left(F_{L}, E\right)\right)=\text { ii-Int }\left(F_{5}, E\right)=\left(F_{5}, E\right)=\left(F_{L}, E\right)=\text { ii-Ext }\left(\text { ii-Ext }\left(F_{L}, E\right)\right) \text {, } \\
& \left(F_{6}, E\right) \tilde{\subseteq}\left(F_{L}, E\right) \text {, ii-Ext }\left(F_{6}, E\right)=\left(F_{3}, E\right) \text {, ii-Ext }\left(F_{L}, E\right)=\left(F_{2}, E\right) \text {, we have } \\
& \mathrm{ii}-\operatorname{Ext}\left(F_{L}, E\right) \tilde{\subseteq} \mathrm{ii}-\operatorname{Ext}\left(F_{6}, E\right) \text {, } \\
& \text { ii-Ext }\left(\left(F_{6}, E\right) \tilde{U}\left(F_{L}, E\right)\right)=\left(F_{2}, E\right) \tilde{\subseteq}\left(F_{3}, E\right)=\mathrm{ii}-\operatorname{Ext}\left(F_{6}, E\right) \tilde{U} \mathrm{ii}-\operatorname{Ext}\left(F_{L}, E\right) \text {, } \\
& \mathrm{ii}-\operatorname{Ext}\left(F_{6}, E\right) \tilde{\bigcap} \mathrm{ii}-\operatorname{Ext}\left(F_{L}, E\right)=\left(F_{2}, E\right) \tilde{\subseteq}\left(F_{3}, E\right)=\mathrm{ii}-\operatorname{Ext}\left(\left(F_{6}, E\right) \tilde{\bigcap}\left(F_{L}, E\right)\right) \text {, } \\
& \mathrm{ii}-\operatorname{Ext}\left(X_{E}\right)=\mathrm{ii}-\operatorname{Int}\left(X_{E} \tilde{\Upsilon}_{E}\right)=\mathrm{ii}-\operatorname{Int}\left(\varnothing_{E}\right)=\varnothing_{E} \text {, } \\
& \mathrm{ii}-\operatorname{Ext}\left(\varnothing_{E}\right)=\mathrm{ii}-\operatorname{Int}\left(X_{E} \tilde{\backslash} \varnothing_{E}\right)=\mathrm{ii}-\operatorname{Int}\left(X_{E}\right)=X_{E} \text {, } \\
& \mathrm{ii}-\operatorname{Ext}\left(X_{E} \tilde{\Upsilon} \mathrm{ii}-\operatorname{Ext}\left(F_{L}, E\right)\right)=\mathrm{ii}-\operatorname{Ext}\left(X_{E} \tilde{\Upsilon}\left(F_{2}, E\right)\right)=\mathrm{ii}-\operatorname{Ext}\left(F_{L}, E\right)=\left(F_{2}, E\right) \text {, } \\
& \text { ii-Int }\left(F_{L}, E\right)=\left(F_{5}, E\right) \simeq \text { ii-Ext }\left(\mathrm{ii}-\operatorname{Ext}\left(F_{L}, E\right)\right) \text {, } \\
& \text { ii-Int }\left(F_{L}, E\right) \tilde{U} \text { ii-Ext }\left(F_{L}, E\right) \tilde{U} \operatorname{ii}-F r\left(F_{L}, E\right)=\left(F_{5}, E\right) \tilde{U}\left(F_{2}, E\right) \tilde{U} \varnothing_{E}=X_{E} \text {, where } \\
& \mathrm{ii}-F r\left(F_{L}, E\right)=\mathrm{ii}-C l\left(F_{L}, E\right) \tilde{\backslash i \mathrm{i}-I n t}\left(F_{L}, E\right)=\left(F_{5}, E\right) \widetilde{(}\left(F_{5}, E\right)=\varnothing_{E} \text {. }
\end{aligned}
$$

\section{Conclusions}

1) Each soft open set is a soft i-open but the converse is not true.

2) Each soft semi-open set is a soft $\mathrm{i}$-open but the converse is not true.

3) Each soft ii-open set is a soft i-open and a soft inter-open set but the converse is not true.

4) Each soft $\alpha$-open set is a soft ii-open set which implies to: each soft $\alpha$-open set is a soft $\mathrm{i}$-open and a soft inter-open set but the converses are not true.

\section{Acknowledgements}

The author is grateful to Prof. Amir A. Mohammed for his valuable remarks.

\section{Conflicts of Interest}

The authors declare no conflicts of interest regarding the publication of this paper.

\section{References}

[1] Molodtsov, D.A. (1999) Soft Set Theory-First Results. Computers \& Mathematics with Applications, 37, 19-31. https://doi.org/10.1016/S0898-1221(99)00056-5

[2] Molodtsov, D.A. (2001) The Description of Dependence with the Help of Soft Sets. Journal of Computer and Systems Sciences International, 40, 977-984.

[3] Aktas, H. and Cagman, N. (2007) Soft Sets and Soft Groups. Information Sciences, 1, 2726-2735. https://doi.org/10.1016/j.ins.2006.12.008

[4] Ali, M.I., Feng, F., Liu, X., Min, W.K. and Shabir, M. (2009) On Some New Operations in Soft Set Theory. Computers \& Mathematics with Applications, 57, 1547-1553. https://doi.org/10.1016/j.camwa.2008.11.009

[5] Maji, P.K., Biswas, R. and Roy, A.R. (2003) Soft Set Theory. Computers \& Mathematics with Applications, 45, 555-562. https://doi.org/10.1016/S0898-1221(03)00016-6

[6] Ayguoglu, A. and Aygun, H. (2011) Some Notes on Soft Topological Spaces. Neural 
Computing and Applications, 21, 113-119.

https://doi.org/10.1007/s00521-011-0722-3

[7] Cagman, N., Karatas, S. and Enginoglu, S. (2011) Soft Topology. Computers \& Mathematics with Applications, 62, 351-358.

https://doi.org/10.1016/j.camwa.2011.05.016

[8] Hussain, S. and Ahmad, B. (2011) Some Properties of Soft Topological Spaces. Computers \& Mathematics with Applications, 62, 4058-4067. https://doi.org/10.1016/j.camwa.2011.09.051

[9] Min, W.K. (2011) A Note on Soft Topological Spaces. Computers \& Mathematics with Applications, 62, 3524-3528. https://doi.org/10.1016/j.camwa.2011.08.068

[10] Peyghan, E., Samadi, B. and Tayebi, A. (2013) About Soft Topological Spaces. Journal of New Results in Science, 2, 60-75.

[11] Shabir, M. and Naz, M. (2011) On Soft Topological Space. Computers and Mathematics with Applications, 61, 1786-1799. https://doi.org/10.1016/j.camwa.2011.02.006

[12] Zorlutuna, I., Akdag, M., Min, W. and Atmaca, S. (2012) Remarks on Soft Topological Spaces. Annals of Fuzzy Mathematics and Informatics, 3, 171-185.

https://www.researchgate.net/profile/Idris_Zorlutuna/publication/296702749_Rema rks_on_Soft_Topological_Spaces/links/56e9570708ae77f87278f7ed/Remarks-on-Sof t-Topological-Spaces.pdf

[13] Askandar, S.W. (2012) The Property of Extended and Non-Extended Topologically for Semi-Open, $\alpha$-Open and I-Open Sets with the Application. M.Sc. Thesis, College of Education, University of Mosul, Mosul.

[14] Njastad, O. (1965) On Some Classes of Nearly Open Sets. Pacific Journal of Mathematics, 15, 961-970. https://doi.org/10.2140/pjm.1965.15.961

[15] Levine, N. (1963) Semi-Open Sets and Semi-Continuity in Topological Space. American Mathematical Monthly, 70, 36-41. https://doi.org/10.1080/00029890.1963.11990039

[16] Chen, B. (2013) Soft Semi-Open Sets and Related Properties in Soft Topological Spaces. Applied Mathematics \& Information Sciences, 7, 287-294. https://doi.org/10.12785/amis/070136

[17] Ilango, G. and Ravindran, M. (2013) On Soft Preopen Sets in Soft Topological Spaces. International Journal of Mathematics Research, 4, 399-409. https://pdfs.semanticscholar.org/eb9b/2ce47c8e1f121bc7514c7488d2b0f9b55d9a.pdf

[18] Mohammed, A.A. and Abdullah, B.S. (2019) II-Open Sets in Topological Spaces. International Mathematics Forum, 14, 41-48. https://doi.org/10.12988/imf.2019.913 http://www.m-hikari.com/imf/imf-2019/1-4-2019/p/mohammedIMF1-4-2019.pdf

[19] El-Sheikh, S. and El-latif, A.A. (2015) Characterization of B-Open Soft Sets in Soft Topological Spaces. Journal of New Theory, 2, 8-18. https://dergipark.org.tr/en/pub/jnt/issue/34483/381064

[20] Ahmad, B. and Kharal, A. (2011) Mappings on Soft Classes. New Mathematics and Natural Computation, 7, 471-481. https://doi.org/10.1142/S1793005711002025

[21] Peyghan, E., Samadi, B. and Tayebi, A. (2014) Some Results Related to Soft Topological Spaces. Journal Facta Universitatis, Series. Mathematics and Informatics, 29, 325-336.

[22] Georgiou, D.N. and Megaritis, A.C. (2014) Soft Set Theory and Topology. Applied General Topology, 15, 93-109. https://doi.org/10.4995/agt.2014.2268 